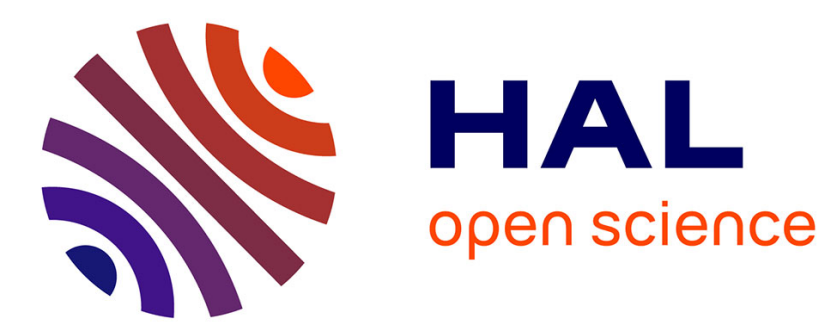

\title{
Changements institutionnels et efficience de l'affrètement au voyage dans le transport fluvial de marchandises
}

Emeric Lendjel, Marianne Fischman

\section{To cite this version:}

Emeric Lendjel, Marianne Fischman. Changements institutionnels et efficience de l'affrètement au voyage dans le transport fluvial de marchandises. 2010. hal-00515244

\section{HAL Id: hal-00515244 \\ https://hal.science/hal-00515244}

Preprint submitted on 6 Sep 2010

HAL is a multi-disciplinary open access archive for the deposit and dissemination of scientific research documents, whether they are published or not. The documents may come from teaching and research institutions in France or abroad, or from public or private research centers.
L'archive ouverte pluridisciplinaire HAL, est destinée au dépôt et à la diffusion de documents scientifiques de niveau recherche, publiés ou non, émanant des établissements d'enseignement et de recherche français ou étrangers, des laboratoires publics ou privés. 


\title{
Changements institutionnels et efficience de l'affrètement au voyage dans le transport fluvial de marchandises
}

\author{
Marianne FISCHMAN \& Emeric LENDJEL ${ }^{1}$

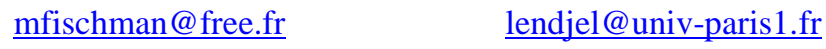 \\ Centre d'Economie de la Sorbonne UMR8174
}

\section{Résumé}

Les données de VNF (2010) montrent une résistance remarquable en France du transport fluvial de marchandises à la récession. Pourtant, une part au moins de la profession est en crise : les artisans bateliers sont aujourd'hui nombreux à être proches du dépôt de bilan. Principaux acteurs du marché de l'affrètement au voyage entièrement libéralisé depuis 2000, les artisans bateliers subissent de plein fouet une baisse des prix résultant d'une concurrence destructrice exacerbée en période de récession. Les coûts de transaction associés au nouvel environnement institutionnel accentuent ce comportement pro-cyclique, préjudiciable à l'ensemble de la profession. L'article s'appuie sur la théorie des coûts de transaction pour évaluer les conditions d'efficience de l'affrètement au voyage avant et après la suppression du «Tour de rôle » et débouche sur des préconisations visant à améliorer l'organisation et l'efficacité de ce segment de marché.

Mots clés : transport fluvial - contrat spot - batellerie artisanale - coûts de transaction

\section{Abstract}

VNF (2010)'s data show a remarkable resilience of inland navigation in France during the recession. Yet at least part of the profession is in crisis: the boatmen are now likely to be close to bankruptcy. Key players in the fully liberalized (since 2000) spot market, small independent barge owners bear the brunt of lower prices resulting from a destructive competition exacerbated in times of recession. Transaction costs associated with this new institutional environment exacerbate this pro-cyclical behavior detrimental to the entire profession. With the help of transaction costs economics, the article assesses the conditions of efficiency of voyage charter in the spot market before and after the abolition of the "Tour de rôle" and lead to recommendations to improve organization and efficiency of this market segment.

Keywords : inland navigation - spot market - small independent barge owners - transaction costs

\footnotetext{
${ }^{1}$ Nous remercions Maurice Bernadet pour ses précieuses remarques sur la version précédente de ce texte présentée sous le titre «La suppression du Tour de rôle et ses conséquences économiques : les nouvelles règles du jeu dans le transport fluvial » au LET lors de la $2^{\text {ème }}$ journée Mobilité Transport et Logistique du 23 juin 2010 à 1'Université de Lyon 2. Nous tenons également à remercier Dany Blanckeman, Didier Carpentier, Olivier Delcourt, Jacques Delhay et Jean-Yves Kerrotret pour l'ensemble des informations qu'ils nous ont fourni.
} 


\section{Introduction}

«La question du tour de rôle et plus fondamentalement celle des modalités de l'affrêtement est depuis longtemps au centre des débats traitant de la compétitivité du transport fluvial »

(Fabart et al., 1986, p. 58).

Il y a dix ans, le $1^{\text {er }}$ janvier 2000, le transport fluvial de marchandises a été entièrement libéralisé avec la suppression d'un mode de régulation administratif du secteur, le «Tour de rôle », laissant aux acteurs (tant du côté de l'offre que de celui de la demande) l'initiative d'organiser leurs échanges. Cette libéralisation n'a concerné qu'une des modalités d'affrètement des navires (l'affrètement au voyage), les deux autres (au tonnage et à temps) échappant à cette régulation administrée.

Dans l'esprit du législateur, le Tour de rôle avait pour but de protéger la batellerie artisanale d'une concurrence destructrice. Au début des années 1930, les artisans bateliers étaient en effet fragilisés par une forte concurrence tarifaire inter - mais aussi et surtout intra modale (française et frontalière), ainsi que par les privilèges des compagnies fluviales (Neiertz, 1999, p. 15). Le Tour de rôle, institué à partir de 1934, a été maintenu jusqu'en 1994, pour être définitivement abrogé en janvier 2001 (loi n²001-43 du 16 janvier 2001) en conformité avec la directive européenne 96/75/CE. La modification de cet environnement institutionnel a instauré de nouvelles règles du jeu.

Avec la disparition de l'organisation selon un Tour de rôle dans le transport fluvial de marchandises, les autorités de tutelles (Commission Européenne et gouvernements nationaux pour l'application de la Directive 96/75/CE du 19 novembre 1996) avaient pour objectif de formuler de nouvelles règles du jeu économique afin d'en améliorer le résultat au regard de l'intérêt collectif. Or, depuis l'introduction du concept de coût de transaction, formulé par Coase en 1937, on sait que l'organisation du marché est coûteuse. Quelle que soit la forme de gouvernance retenue (marché, formes hybrides, hiérarchie), chacune affiche des propriétés économiques alternatives (Williamson, 1993). Les nouvelles règles choisies par les pouvoirs publics ne peuvent pas être hémiplégiques et supposer, en voulant augmenter la productivité globale du secteur, que les coûts de transaction sont nuls (ou très faibles). Si le choix du nouveau mode de coordination est fait en mettant en cause l'importance des coûts d'organisation et le faible niveau de productivité induit par le Tour de rôle ${ }^{2}$, la mise en place de la libéralisation ne peut se faire sans tenir compte des coûts de transaction qui lui sont associés. L'objet de ce travail est de mettre en lumière l'importance des coûts de transaction (Williamson, 1985 ; 1996) supportés dans le fluvial et de montrer qu'ils constituent une entrave au développement de ce secteur d'activité, en particulier pour la batellerie artisanale.

L'affrètement au voyage étant auparavant la seule modalité d'affrètement soumise au Tour de rôle, les deux autres modalités (au tonnage ou à temps) ne seront pas abordées dans ce travail. Cette modalité d'affrètement exclut les transports organisés autour de rotations régulières, comme le transport conteneurisé, le transport de marchandises impliquant des actifs spécifiques (le ciment, les produits pétroliers, les voitures). L'affrètement au voyage est une modalité d'affrètement qui n'engage pas les parties au-delà du voyage contracté, même si les relations entre elles peuvent être assez fréquentes. L'irrégularité de cet affrètement conduit en général la batellerie industrielle à s'en désintéresser. Sauf en cas de crise économique, cette modalité d'affrètement ne concerne que la batellerie artisanale.

Pour l'analyser, nous commencerons dans la première section par définir la transaction de transport fluvial pour montrer que la structure de gouvernance de l'affrètement au voyage est alignée sur les attributs de cette transaction. Mais, cette structure de gouvernance engendre

\footnotetext{
${ }^{2}$ Grégoire, 1983 ; Fabart et al, 1986 ; Neiertz, 1999.
} 
des coûts. Les deuxième et troisième sections s'attacheront à les déterminer dans des environnements institutionnels voisins. Nous rappellerons tout d'abord que l'absence de réglementation caractérisant la période libérale au tournant du $20^{\text {ème }}$ siècle engendrait d'importants coûts de transaction. Nous examinerons ensuite les coûts de transactions rencontrés dans l'environnement institutionnel actuel. Cette recherche débouche sur des recommandations pour le secteur. Nous montrerons que des mesures simples peuvent être prises tant par les acteurs que par les pouvoirs publics pour améliorer l'organisation et l'efficacité sur le segment de marché considéré. C'est à ce prix en effet que l'on évitera d'assister au retour d'une forme de concurrence destructrice qui n'est pas sans rappeler celle des années trente, qui n'est probablement pas étrangère à la pire crise économique connue depuis 1929 aux dires de nombreux commentateurs.

\section{La chaîne transactionnelle}

Williamson définit la transaction comme le transfert de biens ou services entre interfaces technologiquement séparables (Williamson, 1985, p. 1). Le transport à l'aide d'une péniche constitue une unité de production technologiquement séparable. La manutention portuaire en constitue une autre ; le pré- et post-acheminement routier une troisième... Le transport fluvial de marchandises imbrique ainsi une multitude de transactions articulées dans une ou plusieurs chaînes transactionnelles. Ces chaînes peuvent être plus ou moins intégrées selon les différentes modalités contractuelles retenues par les acteurs, tissant une trame complexe de relations entre les acteurs.

La complexité du transport fluvial de marchandises, sous le regard de l'analyse transactionnelle, nécessite de décrire au préalable la (voire des) chaînes transactionnelles. La chaîne transactionnelle du transport fluvial de marchandises implique en général un pré- et post-acheminement routier, même pour le transport de marchandises en vrac, ainsi que de la manutention portuaire pour charger et décharger les marchandises sur les ports. L'une des chaînes contractuelles possibles structurant ces transactions dans le fluvial est celle décrite dans le graphique ci-dessous.

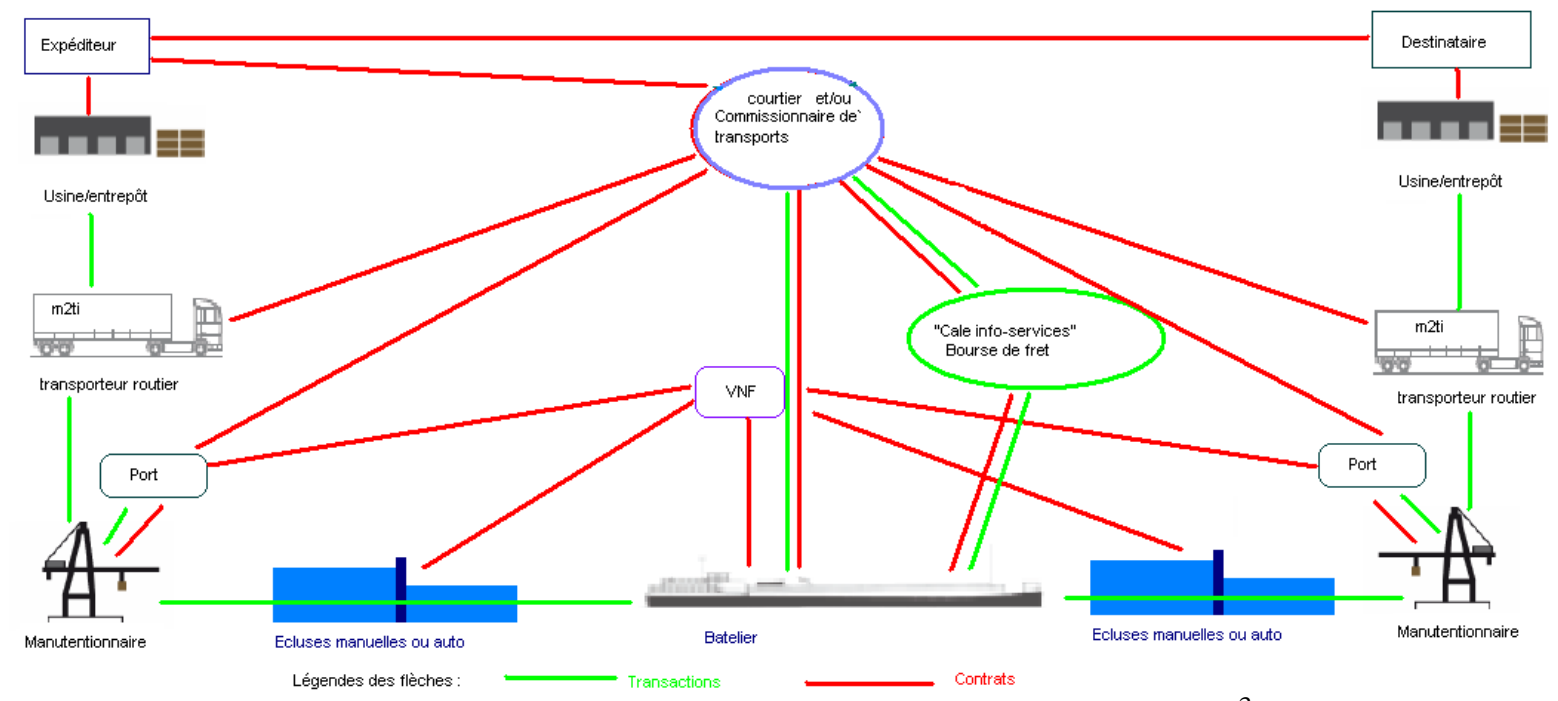

Comme $90 \%$ du fluvial se vend «quai à quai », sans prestations annexes ${ }^{3}$, le périmètre de la transaction que nous retiendrons ici comprend les sous-transactions de premier rang du transporteur, soit le triptyque transporteur-manutentionnaire-courtier/commissionnaire. Dans le cadre d'affrètements au voyage, la structure de gouvernance est par définition celle du

\footnotetext{
${ }^{3}$ Interview de J.Y. Kerrotret, Logistra.
} 
marché ${ }^{4}$. Le batelier est en relation contractuelle avec un courtier qui souvent se confond avec un commissionnaire de transport ${ }^{5}$. L'expéditeur ne s'adresse pas forcément au même courtier. Soufflet Rouen, par exemple, a fait transporter en 2007 et 2008 ses céréales par le même artisan batelier, mais à chaque fois par l'intermédiation d'un affréteur (courtier/commissionnaire) différent. Le graphique est donc ici volontairement simplifié.

Traditionnellement, la théorie des coûts de transaction stipule que la structure de gouvernance (hiérarchie, hybride, marché) doit être alignée sur les attributs (degré de spécificité d'actifs, fréquence, incertitude) de la transaction (Williamson, 1985, 1996). Les attributs correspondant au choix d'affrètement au voyage devraient logiquement être un degré moyen de spécificité d'actif, une fréquence moyenne de transaction et une incertitude moyenne.

\section{Les actifs}

Parmi les différentes spécificités d'actif (physique, dédié, humain, réputation, site) (Williamson, 1985; 1989), trois semblent particulièrement importantes pour notre compréhension du secteur fluvial.

Le réseau navigable est un actif physique hautement spécifique dont la qualité (interconnexions offertes, tirant d'eau et tirant d'air, débit, heures de fonctionnement des écluses, etc.) est déterminante sur l'efficacité économique des transports fluviaux (Allam et Lendjel, 2009). Il comporte également une spécificité de site importante en raison des différents gabarits qui le composent. Les tronçons à grands gabarits (bassins de la Seine, du Rhin et du Rhône) ne sont en effet pas interconnectés entre eux, sauf par les canaux de classe I (Freycinet). Le redéploiement des navires sur des bassins différents engendre des coûts importants. A l'inverse, les automoteurs Freycinet sont susceptibles de circuler sur tout le réseau européen de classe I jusqu'à la mer noire s'ils le souhaitent. A cette enseigne, ces actifs n'ont donc qu'un degré moyen de spécificité si l'on tient compte des coûts de repositionnement.

La transaction de transport fluvial implique des compétences humaines spécifiques, tant en amont (commerciales, organisationnelles, etc.) qu'en aval (liée à la prestation de transport proprement dite).

Du côté de l'affréteur, les courtiers/commissionnaires de transport sont des personnes sédentaires aux compétences les plus spécifiques. Elles ont pour mission de mettre en adéquation une capacité dynamique de transport (disponible au temps $t$ pour une période donnée, au départ d'un port $x$ pour un port $y)^{6}$ avec une demande émanant d'un chargeur en optimisant le taux de remplissage du navire. Leur raison d'être (et leur rémunération) réside dans leur capacité à centraliser des informations multiples dans sa zone de chalandise (dont certaines de nature qualitative, comme la fiabilité du transporteur, son expérience, les caractéristiques de sa cale, etc.) et à rendre possible la transaction. Un courtier occupe une fonction analogue à celle d'un responsable d'exploitation dans une entreprise de transport routier, mais sans avoir à supporter le même niveau de situations non-routinières (Allam et Lendjel, 2009). Leurs compétences sont de nature commerciales, techniques,

\footnotetext{
${ }^{4}$ Les formes hybrides et intégrées sont contractualisées par des affrètements au tonnage et des affrètements à temps.

${ }^{5}$ Leurs fonctions et leur responsabilité contractuelles sont pourtant théoriquement différentes. Le courtier se contente de mettre en relation un chargeur (expéditeur ou destinataire) avec le transporteur, moyennant une commission payée par le transporteur; le commissionnaire de transport se voit confier par le chargeur la responsabilité d'un transport de marchandises à un prix donné, qu'il sous-traite à un transporteur à un prix par définition inférieur (Lamy, 2009).

${ }^{6}$ La capacité dynamique de transport d'un navire dépend de sa capacité statique de transport (ou son «port en lourd ») et de sa vitesse de déplacement. Plus sa vitesse augmente, plus sa capacité de transport augmente durant une période donnée.
} 
organisationnelles mais également humaines et diplomatiques (en particulier vis-à-vis de l'indépendance des artisans bateliers). Les compétences relationnelles jouent ici un rôle central dans l'efficacité du dispositif. La relation de confiance avec les bateliers est indispensable pour travailler efficacement. Ces « ressources intangibles [...] réduisent les coûts de communication, de négociation et de résolution de tout type de problèmes de coordination et de contrats » (Arrunada et al., 1998, p. 9).

La spécificité des actifs humains est également forte du côté des «pénichiens » (Le Sueur, 1986). Elle consiste bien évidemment dans la conduite du navire lui-même et des règles de navigation. Mais elle comprend également toute une série de connaissances moins formelles : celle des rivières et canaux, de la profondeur des fonds, de la hauteur des ponts, de l'état des voies navigables ${ }^{7}$, de la largeur et de la longueur des écluses, de leur état de fonctionnement, de la maîtrise de la navigation en cas de difficultés (crue, faible tirant d'eau... ). Souvent, les bateliers ont une parfaite connaissance de leur bassin de navigation et doivent faire appel aux connaissances d'autres bateliers lorsqu'ils s'aventurent sur une autre voie d'eau. C'est cette connaissance qui permet aux bateliers de maîtriser leur temps de navigation avec une très grande exactitude, la ponctualité étant un des atouts du transport fluvial de marchandises. Les bateliers doivent également maîtriser le chargement du navire, connaître les pratiques dans les ports tant fluviaux que maritimes dans lesquels ils chargent et déchargent, sans compter leur connaissance aigüe de la géographie fluviale (distance, lieux...).

\section{Fréquence et incertitude}

Malgré leur degré élevé de spécificité, les relations entre le courtier et le pénichien sont trop peu fréquentes pour justifier une intégration verticale. Au mieux, un artisan batelier sur du gabarit Freycinet réalise en effet environ une trentaine de transports par an. La lenteur de ce mode de transport ainsi que les temps d'attentes nécessaires pour charger et décharger les marchandises (délais de planche, surestaries) ne permettent pas une fréquence élevée des transactions. Les carnets de voyage fournis par un artisan batelier interviewé témoignent en effet, pour les années 2007 et 2008 (donc sur un volume total de 61 transactions), une durée moyenne du contrat au voyage de 6 jours.

Relevons, par ailleurs, que la lenteur de ce mode de transport est incompatible avec un degré élevé de spécificité temporelle (Pirrong, 1993). La valeur des marchandises généralement transportées par voie fluviale reste assez insensible aux temps (de stockage, comme de transport) et ne nécessite pas un «ajustement fin entre la livraison et l'usage d'un bien » (Glachant et Hiroux, 2010). Le risque de comportements opportunistes est ici faible ${ }^{8}$.

En revanche, une source d'incertitude, de nature comportementale ou institutionnelle (Masten et Saussier, 2002 ; Finon et Perez, 2007), mérite d'être signalée ici. L'incertitude comportementale renvoie aux interactions entre les agents et aux prescriptions de régulation sur les différents maillons du transport fluvial (Glachant et Hiroux, 2010). Le transport sur la voie d'eau comporte deux sous-transactions qui, contrairement à l'image qu'en donne la péniche, ne sont pas forcément intégrées. Historiquement, si l'on considère les 150 dernières années de transport fluvial, c'est même le contraire qui domine trois quart du temps. La traction/propulsion et le transport sont en effet deux transactions à interfaces technologiquement séparables. Au $19^{\text {ème }}$ siècle et jusqu'au premier tiers du $20^{\text {ème }}$ siècle, le halage est distinct du transport proprement dit et fait l'objet de contrats marchands entre le « pénichien » et les « longs jours » (Le Sueur, 1986, 2004). Il en va de même avec les toueurs,

\footnotetext{
${ }^{7}$ L'état de l'infrastructure est d'autant plus mauvais que le gabarit est petit, le réseau Freycinet étant laissé à l'abandon de l'avis même de nombreux spécialistes (Damien, 2009, p. 352).

${ }^{8}$ Sur les années 2007 et 2008, l'artisan batelier interviewé ne mentionne qu'un seul contrat annulé sur 62 transactions observées.
} 
bateau-treuil à vapeur tractant une dizaine de péniches enchaînées les unes aux autres. De même, à partir des années 1960, les pousseurs sont dissociés des barges qu'ils propulsent et font l'objet de transactions distinctes. Cette souplesse transactionnelle procure d'importants gains de productivité en évitant au pousseur d'avoir à attendre le chargement/déchargement du fret. Mais pour exploiter pleinement cette faculté et amortir le coût important d'un pousseur, les rotations du pousseur impliquent une programmation de long terme des trajets et une coordination des flux incompatibles avec le marché. La structure de gouvernance est alors logiquement hybride ou intégrée.

Par contraste, l'automoteur de type Freycinet internalise la propulsion principalement pour trois raisons : 1/ la séparation entre le halage et le transport était historiquement source de dépendances mutuelles et de comportements opportunistes (Le Sueur, 1986, pp. 50-52). L'intégration de la propulsion a permis de supprimer ce risque. 2/ Le coût important d'un pousseur (approximativement dix fois plus élevé qu'un Freycinet) freine aujourd'hui les velléités d'investissements des artisans bateliers. 3/ Surtout, le gabarit du réseau emprunté contraint la taille maximale des navires. En effet, la longueur maximale admise dans les écluses du réseau Freycinet est de 38,5 mètres. L'optimisation économique de ce gabarit implique donc l'intégration de la propulsion sur les péniches souhaitant emprunter ce réseau. En contrepartie de cette nécessaire intégration, les pénichiens sont obligés de supporter les temps d'attentes au chargement et déchargement de leurs marchandises. Ces temps introduisent des aléas d'exploitation importants favorisant les contrats au voyage.

L'alignement de la structure de gouvernance (contrat au voyage) sur les attributs de la transaction semble donc bien être assuré (Williamson, 1985). Reste que l'ajustement de l'offre et de la demande nécessite la mise en place d'un mécanisme de coordination qui peut prendre plusieurs formes et supporter des coûts de transaction variables.

\section{Une lecture transactionnelle de l'histoire des modalités de l'affrètement au voyage}

L'Histoire de la batellerie nous montre que le fonctionnement du marché engendre d'importants coûts de transaction affectant l'organisation du halage ( $c f$. Le Sueur, 1986, pp. 50-53), d'une part, et celle de l'affrètement, de l'autre. L'inorganisation qui caractérise le service de halage indispensable jusqu'à l'essor des automoteurs dans les années 30 est problématique, en raison de l'intensité du trafic sur certains tronçons ${ }^{9}$. La généralisation des automoteurs, dans les années trente, en a toutefois eu raison. Elle correspond à une internalisation de ce service par les transporteurs, rendue possible par une innovation technologique et justifiée par l'accroissement de l'efficience de la transaction de transport fluvial qu'elle génère. Ce n'est en revanche pas le cas de l'organisation de l'affrètement, dont les difficultés continuent de poser problème aujourd'hui. C'est pourquoi leur analyse (2.1) et surtout celle des solutions successives (2.2) qui ont été proposées pour tenter d'y remédier avec plus ou moins de succès (2.3.) jusqu'à nos jours, nous est précieuse. Elle éclaire les choix à venir pour l'avenir de la profession et de possibles préconisations.

\footnotetext{
${ }^{9}$ C'est ainsi le cas dans les $20 \mathrm{~km}$ du bief de partage du canal de Saint-Quentin sur lequel le trafic s'est fortement accru entre 1850 et 1913 (Pugin, 1982, p. 49-51)
} 


\subsection{La diversité des systèmes d'affrètement libres et privés (1886 - 1904)}

S'il est vrai que la libéralisation dans le transport fluvial de marchandises a marqué une rupture importante au début des années 2000 en France, le travail des historiens nous rappelle que la batellerie n'en est pas à sa première adaptation réglementaire ${ }^{10}$. On peut ainsi dégager quatre périodes :

- jusqu'au XVIII ${ }^{\text {ème }}$ siècle, où s'impose la volonté d'uniformiser, de rationaliser et d'harmoniser la législation de la navigation fluviale;

- les XVIII ${ }^{\text {ème }}$ et XIX ${ }^{\text {ème }}$ siècles, marqués par la mise en place progressive d'une politique libérale dans le transport fluvial de marchandises, comme dans maints d'autres domaines en France ;

- la première partie du $\mathrm{XX}^{\text {ème }}$ siècle caractérisée par un retour de plus en plus prégnant de l'Etat ;

- et enfin, de 1950 à nos jours, constituée d'une nouvelle période d'adaptation de la réglementation puis de déréglementation.

L'Ordonnance des Eaux et Forêts de 1669 constitue en effet le socle institutionnel de référence aux multiples décisions à venir (Le Sueur, 1993, p. 66). Les textes se superposent rapidement et donnent lieu en un siècle à un impressionnant corpus dans lequel se trouve déjà une répartition des voyages au tour de rôle, dans les corporations de bateliers du Nord par exemple (idem, p. 67) ${ }^{11}$.

Mais le temps des Economistes sonne le glas des corporations et souffle le vent de la liberté du commerce (Fischman, 1998). Dans le domaine de la voie d'eau, cela se traduit par la dissolution des corporations en 1791

«et la fin de leurs privilèges et monopoles. Le législateur cherche à donner la plus grande liberté possible à la circulation fluviale, et reconnaît le droit d'entreprise et de transport par eau. En elle-même, l'activité commerciale par voie d'eau ne fait l'objet d'aucun texte réglementaire spécifique. Le Code civil et le Code du commerce sont bientôt les seules références. [...] En revanche, les dispositions de 1669 destinées à faciliter la navigation sont confirmées et renforcées en maintes occasions » (Le Sueur, 1993, p. 68).

A partir de cette époque, les contrats de transports sont privés ${ }^{12}$ et les transactions, absolument libres. En revanche, il faut attendre la loi Guyot de 1880 pour que soient supprimés tous les impôts spécifiques du transport fluvial, qui utilise alors désormais « gratuitement fleuves, rivières et canaux » (idem., p. 71). Cette situation perdure jusqu'au début du $\mathrm{XX}^{\mathrm{e}}$ siècle.

$\mathrm{Au}$ cours de ces années de grande liberté économique, de nombreuses transformations marquent le transport par voie d'eau et des difficultés apparaissent. Ces transformations affectent le réseau, le marché (espace géographique et marchandises transportées) de la voie d'eau ainsi que la profession (Le Sueur, 1982 ; 1986 ; Gerritsen, 1983). Jusqu'au tout début du $\mathrm{XX}^{\mathrm{e}}$ siècle, les principales difficultés pour réaliser les transactions de transport fluvial de marchandises portent sur

\footnotetext{
${ }^{10} \mathrm{La}$ réglementation est triple. Elle est relative aux voies navigables, à la police de navigation et aux contrats de transports. C'est cette dernière qui retient essentiellement notre attention ici.

${ }^{11}$ Le tour de rôle « repose sur le respect des dates de mise à quai pour le batelier devenu disponible, celui des dates d'enlèvement pour le fret attribué par la bourse régionale et les droits de tous les bateliers à être appelés dans l'ordre de ces dates. Il existe également en RFA, aux Pays-Bas et en Belgique mais sur des bases différentes. Il s'agit là d'une règle interne de redistribution du fret au sein des coopératives » (Neiertz, 1999, p. 588).

${ }^{12}$ Le code du commerce de 1807 est la seule référence officielle jusqu'à la fin du XIX ${ }^{\mathrm{e}}$ siècle (Le Sueur, 2005, vol. 2, p. 89).
} 
- l'information (relative à l'offre et à la demande de transport), notamment sa faible disponibilité et son incomplétude : avec une multitude de transporteurs et de chargeurs géographiquement éparpillés entre les divers sites de chargement et de déchargement des marchandises, comment savoir quelle est la cale disponible ? A quelle date ? comment connaître le sérieux et le professionnalisme du transporteur ? le besoin du chargeur? son degré de fiabilité ? etc. Or, ces deux caractéristiques sont génératrices d'importants coûts de recherche et de production pour les acteurs: coûts des déplacements ou d'attente à vide pour le transporteur, coûts de stockage pour les chargeurs, de retard dans les livraisons de marchandises, coûts de la mise en place d'un réseau social important, frais de publicité... pourtant indispensables pour parvenir à effectuer les transactions (Ménard, 1997);

- l'intensification de la demande de transport (liée au développement des Mines en particulier) et donc du nombre de transports à exécuter mais aussi de leur fréquence. La fréquence accrue des prestations de transport n'accroît pas simplement les coûts liés à la production et la recherche d'information, mentionnés précédemment. Elle génère également des coûts de transaction supplémentaires (temps de négociation des conditions de transport pour chaque contrat) conséquents dès lors que les contrats de transport restent au voyage et n'ont donc pas le caractère régulier systémique des contrats au temps ou à la tonne voire de la mise en place des lignes régulières. Il est alors clair que la réduction de ces coûts ne peut être obtenue que par la modification des contrats, quels que soient les systèmes d'affrètement considérés;

- la fixation du prix (qui rend compatible les plans a priori divergents des acteurs : les chargeurs, les transporteurs et les courtiers). Non seulement le prix du transport fluvial de marchandises est particulièrement instable à cette époque, mais surtout il y a une grande incertitude sur la fiabilité de l'information qu'il transmet : le prix du transport comprend-il les temps d'attente pour le chargement et le déchargement ? Inclut-il la responsabilité du transporteur, en particulier en cas de vol ou de détérioration de marchandises ? Reflète-t-il l'état exact du niveau de l'offre et de la demande de transport ou la seule méconnaissance qu'en ont les acteurs ? etc. Toutes ces interrogations ouvrent la voie aux contestations des bateliers. Ils jugent le prix trop bas et les frais trop élevés - au point de rendre souvent problématique la réalisation des transactions, comme en témoignent les nombreux incidents qui se produisent dès la fin du siècle, et surtout les grèves de 1904 et la persistance des conflits sociaux jusqu'à la création de l'Office national de Navigation (l'O.N.N.) en 1912.

Pour tenter de remédier à ces problèmes, divers systèmes d'affrètement sont expérimentés entre 1886 et 1904 et coexistent parfois (Le Sueur, 1986, pp. 42-44, 2005, pp. 89-101) ${ }^{13}$. Ils peuvent être regroupés en deux catégories : les bourses et les marchés intermédiés.

Les bourses comprennent $1 /$ le contact direct entre bateliers et chargeurs dans les estaminets. 2/ les bureaux d'affrètements mis en place par les Compagnies Minières de manière dispersée dans toute la région minière ; 3 / les premiers marchés d'affrètement sur les places publiques créés par les employés des Mines et des maisons de transport ; 4/ la Chambre d'affrètement mise en place par le comité des houillères en juillet 1904; 5/ la Société coopérative de transports fondée en décembre 1904.

Les marchés intermédiés sont 1/ la généralisation des marchés d'affrètement sur les places publiques avec de véritables intermédiaires entre l'offre et la demande de transport - les

\footnotetext{
${ }^{13}$ Quel que soit le système d'affrètement retenu, celui-ci doit en effet résoudre la quadrature du cercle suivante : du côté des chargeurs, leur assurer une disponibilité de cale répondant à leurs besoins, en leur laissant la faculté de choisir le transporteur, au coût le plus bas possible ; du côté des transporteurs, leur limiter les parcours sans charges et les attentes non rémunérées, en leur laissant la faculté de choisir les transports qu'ils souhaitent effectuer au prix le plus élevé possible.
} 
courtiers, affréteurs professionnels indépendants (comme la maison Delegrange à Douai) dont le nombre et l'importance croissent rapidement (puisque $90 \%$ des bateaux affrétés au début du siècle, le seront par ces intermédiaires) ; 2/ les bureaux d'affrètement mis en place à Charleroi par l'Agence d'Affrètement de la Société du canal de la Sambre à l'Oise à partir de 1886 ou issus d'autres initiatives privées ; 3/ les bureaux de tour à Dunkerque et à Calais créés fin 1904, dans la lignée du programme élaboré par le Groupement Général de la Batellerie consistant en «la prise en mains par les bateliers eux-mêmes de l'exploitation commerciale des voies navigables » mais organisés dans la pratique par des courtiers (Le Sueur, 1986, p. 43).

L'intérêt de ces systèmes est pluriel. Ils permettent d'apporter des réponses aux problèmes posés par les coûts de production et de recherche de l'information, accrus par l'accroissement des fréquences de la transaction de transport. En revanche, les difficultés restent presque entières en ce qui concerne les prix.

\subsection{Apports des systèmes d'affrètement mis en place jusqu'en 1904}

En limitant la dispersion des lieux de rencontre des acteurs, les systèmes d'affrètement ci-dessus permettent tous de centraliser les informations et de les donner plus rapidement. Ils facilitent ainsi la coordination entre les acteurs et par conséquent évitent de retarder les transactions. Il est clair que plus la centralisation est forte, moindre est la dispersion et plus sont évités les coûteux retards dans la réalisation de la transaction de transport. De ce point de vue, les marchés intermédiés (et dans une moindre mesure la Chambre d'affrètement des Houillères et la Société coopérative de transport par eau dès lors que son exploitation s'élargit à un grand nombre de bateaux ${ }^{14}$ ) sont plus efficaces que les bourses. Cela explique sans doute qu'ils aient perduré jusqu'à nos jours, sous une forme ou sous une autre.

On notera tout particulièrement l'existence d'organisations dans le système des bourses. Elles sont créées par regroupement des acteurs, transporteurs (les bureaux de tour de Dunkerque et de Calais ou tout au moins tels qu'ils étaient définis dans le projet du Groupement général de la Batellerie) ou affréteurs (la Chambre des Houillères). Elles internalisent la fonction commerciale ${ }^{15}$ rendue d'autant plus performante qu'elle mutualise les informations des membres du groupe.

Mais il faut bien entendu souligner le rôle central des courtiers (et dans une moindre mesure de l'Agence d'Affrètement de Charleroi), à propos de cette fonction commerciale dans laquelle ils sont spécialisés, disposant d'un réseau d'informations très efficace.

C'est alors dire que ces organisations dans les bourses, comme les sociétés de courtage dans les marchés intermédiés, jouent un rôle important dans la centralisation de l'information et la limitation de la dispersion des sources d'information qui en résulte, accélérant ainsi sa mise à disposition et réduisant par voie de conséquence les coûts liés à son obtention par rapport aux situations où ces organisations font défaut.

Mais c'est aussi souligner que toutes deux permettent d'améliorer la quantité et la qualité de l'information disponible, c'est-à-dire de limiter l'incomplétude de l'information, par la mutualisation des informations ou par la spécialisation dans la recherche d'informations qui les caractérisent respectivement.

A ce titre, l'émergence des intermédiaires que sont les courtiers joue un rôle central pour remédier aux asymétries entre participants aux échanges (dans l'information détenue et

\footnotetext{
${ }^{14}$ Ce qui est progressivement le cas puisqu'elle exploite une centaine de bateaux en 1909 contre une vingtaine en 1904 (Le Sueur, 2005, p. 116).

15 Cette fonction consiste à réaliser 1/ la publicité relative à l'offre et à la demande de transport (soit la production et la mise à disposition de ces informations à l'ensemble des acteurs), 2/ la négociation voire la rédaction des contrats, 3/ la répartition des voyages entre les transporteurs et, éventuellement, 4/ la mise en place de mécanisme de stabilisation des prix.
} 
dans leur capacité à l'acquérir). Deux raisons à cela. 1/ Parce qu'ils sont des tiers rémunérés sur les transactions réalisées entre les parties. Ils ont donc tout intérêt à ce que les transactions aient lieu dans l'intérêt des deux parties transporteurs et chargeurs pour qu'elle se reproduise. 2/ Car la spécialisation que constitue leur métier fait d'eux les plus compétents dans le domaine, compétence émulée par la concurrence qu'ils se livrent entre eux.

L'importance de ce rôle explique que les transporteurs aient boudé la chambre des Houillères en 1904. Elle explique aussi pourquoi les bureaux de tour de Dunkerque ont perdu un grand nombre de transports après $1909^{16}$, sous la pression des chargeurs. Ceux-ci souhaitent en effet pouvoir connaître à l'avance le batelier avec lequel ils traitent et le choisir. Or, bien qu'organisé par les courtiers, le tour de rôle ne permettait pas à ceux-ci de remplir pleinement leur fonction d'intermédiation, et donc de choisir les transporteurs répondant au mieux aux besoins des chargeurs puisque seuls les bateliers étaient maîtres du choix de prendre, ou non, les voyages affichés par les courtiers.

Pourtant, c'est le coût supplémentaire généré par l'intermédiation du courtier qui explique historiquement la naissance d'organisations de transporteurs ou de chargeurs, l'éviction du courtier permettant des prix plus bas qu'un marché avec intermédiaires.

\subsection{Un environnement institutionnel générant d'importants coûts de transaction}

Le point d'achoppement essentiel de tous ces systèmes est le prix. Outre la question de sa stabilité, c'est l'incertitude sur l'information que véhicule le prix qui pose problème pour la réalisation des transactions.

\section{Absence contrat type}

L'absence de contrat type - par exemple stipulant la nature et la quantité de la marchandise transportée, les dates et les délais de chargement/déchargement, les frais divers directement associés au transport à réaliser (commission d'affrètement en particulier), les montants des indemnités éventuelles en cas de non respect des éléments du contrat - engendre des coûts de négociation importants et des coûts de règlement de litiges postérieurs accrus. En découlent de graves dysfonctionnements dans le déroulement même de la prestation et dans la répartition des frais liés à ces dysfonctionnements notamment les frais liés aux retards, à l'attente pour charger et décharger les marchandises, mais aussi les frais liés à la différence entre la masse de marchandises à charger, effectivement en cale au chargement, puis au déchargement.

«Dans les contrats fleurissent des clauses particulièrement difficiles à respecter par les pénichiens. Par exemple, certains accords de transport ne sont même pas reconnus par les mines qui peuvent laisser en attente le bateau pendant des semaines ! Le batelier est souvent rendu responsable de tout retard dans la livraison de sa marchandise, quelle qu'en soit la raison ! Arrivé à destination, il peut être contraint de décharger son bateau de jour comme de nuit, au bon vouloir du client. S'il demande une avance de fret pour pouvoir payer les premiers frais de voyage, il se voit appliquer des taux usuraires... » (Le Sueur, 2005, p. 103).

De ce point de vue, le contact direct entre bateliers et chargeurs dans les estaminets ou entre bateliers et courtiers sur les marchés d'affrètement généralisés sur les places publiques constitue un mode d'organisation particulièrement efficient. Car il permet de limiter les coûts de transaction liés aux asymétries d'information par l'établissement de relations contractuelles, fussent-elles informelles ${ }^{17}$, sur la base de relations de confiance entre les acteurs. L'importance de ces coûts et l'incapacité des autres systèmes à résoudre ce problème

\footnotetext{
${ }^{16}$ C'est la date de la renégociation de l'accord prévu pour 5 ans lors de sa signature en 1904.

${ }^{17} \mathrm{Ne}$ serait-ce qu'en raison du faible niveau d'instruction des bateliers, souvent illettrés à cette époque.
} 
de manière plus efficiente expliquent pourquoi ce type de pratique fondée sur la confiance perdure jusqu'à nos jours.

Dans le cas d'un contact direct avec le client, il s'est développé tout au long du XX $\mathrm{X}^{\mathrm{e}}$ siècle (Le Sueur, 2005, p. 90). Il présente en outre l'avantage de fournir du fret avec régularité, régularité généralement obtenue au prix d'un taux de frêt inférieur à celui du marché mais permettant de bénéficier de revenus quasi-fixes eux-mêmes réguliers ${ }^{18}$. Aujourd'hui, cette relation de confiance perdure et ce alors même que les contrats types existent. Cela vient de ce que la formalisation par écrit des contrats d'affrètement n'est toujours pas systématique (comme en témoigne aujourd'hui le protocole d'accord récemment signé entre plusieurs courtiers, le CAF, la Glissoire et la CNBA, stipulant «l'obligation de formaliser par écrit les contrats d'affrètement dans le respect des textes »). En revanche, cette relation de confiance s'établit désormais essentiellement entre transporteurs et courtiers en raison de la facturation à 60 jours, par le chargeur, contre 10-15 jours, par le courtier. Enfin, l'autre intérêt de cette régularité du transport est qu'elle permet aux acteurs de diminuer leurs coûts de recherche d'information sur la cale disponible et la demande de transport.

Notons toutefois que le projet du Groupement Général de la Batellerie de négocier tous les contrats et de les rédiger conformément à un texte type cherchait bien à solutionner le problème ici soulevé. C'est aussi un des points de l'accord accompagnant la création de bureau de tour à Dunkerque à la fin de l'année 1904 : un contrat unique d'affrètement est reconnu, fixant de surcroît la commission d'affrètement à 5\% du total, ainsi que les délais de chargement/déchargement de marchandises et les indemnités à payer en cas de dépassement (idem, p. 112). Des éléments en ce sens avaient déjà également été défendus par un ensemble de courtiers ainsi que par la Chambre des Houillères, suite aux premiers incidents de la fin du $\mathrm{XIX}^{\mathrm{è}}$ siècle (ibid., p. 104). Pour limiter les temps d'immobilisations des bateaux, les premiers réclamaient en effet un système de bons de chargement établis à la signature des contrats. Quant aux seconds, ils proposaient de préciser les délais d'enlèvement des charbons. Mais jusqu'à la Première Guerre Mondiale, les règles et indemnités afférentes relatives au temps de mise à disposition des bateaux pour les opérations de manutention, à savoir les jours de planche, les staries et les surestaries ${ }^{19}$ ne sont pas clairement établies. Ces dernières sont même considérées comme un loyer versé, soit « une somme forfaitaire calculée à la journée » (Le Sueur, 2005, p. 96), nullement comme de véritables dommages et intérêts ${ }^{20}$.

Soulignons, enfin, que l'on retrouve (cf. 2.2) le rôle central des organisations (regroupant un ensemble d'acteurs sinon atomisés) dans la mise en place de procédures réduisant les coûts liés aux transactions - ici la mise en place de règles et de conventions.

Or, celles-ci sont essentielles pour résoudre les difficultés mentionnées, c'est-à-dire pour connaître avec exactitude la nature des prestations à effectuer, la responsabilité de chacun, et déterminer le degré d'engagement de chacune des parties (en particulier le temps d'attente au-delà duquel le batelier n'est plus astreint à l'immobilité). C'est tout particulièrement le cas de la définition précise des staries et des surestaries. Celle-ci joue en effet un rôle incitatif fort tant du côté des transporteurs que de celui des chargeurs pour qu'ils

\footnotetext{
${ }^{18}$ Cette baisse du taux de fret s'explique par la disparition avec des contrats réguliers des coûts de transaction supplémentaires générés par la fréquence de la transaction, ie le temps de négociation des conditions de transport pour chaque contrat.

${ }_{19}^{19}$ «pour assurer le chargement ou le déchargement d'un bateau mis à la disposition d'un client pour un voyage, on accorde à ce dernier un délai considéré comme normal. Ce temps d'immobilisation à quai correspond à ce que les mariniers appellent de manière imagée, les jours de planche, puisque le gamberet, la planche, est en place en permanence » (Le Sueur, 2005, p. 97). Ce sont les staries, dans la terminologie des courtiers et des chargeurs. Au-delà de ce délai normal, le temps passé est indemnisé par les surestaries.

${ }^{20}$ Cette situation est propre à la France. La loi fixe les délais et les indemnités sont définies proportionnellement au tonnage en Allemagne depuis 1895, en Hollande depuis 1898 et en Belgique depuis 1900 (Le Sueur, 2005, p. $116)$.
} 
mettent tout en oeuvre en vue d'accélérer les rotations des bateaux (temps de transport, temps de transbordement, date de mise à quai, de livraison, temps d'attente normal à quai et temps d'immobilisation à vide maximum).

\section{L'anarchie des intermédiaires}

En l'absence d'application de la réglementation de la profession des intermédiaires, et donc par excellence du courtier ${ }^{21}$, « les abus se multiplient et la pratique de la sur-commission [ou ristournes, correspondant au prix demandé par les courtiers aux bateliers, en sus de leur commission, pour leur obtenir un voyage. Cette somme est défalquée du frêt payé par le chargeur, l'incitant à recourir au courtier pratiquant cette ristourne] se généralise » (Le Sueur, 1986, p. 42) ${ }^{22}$.

Surtout, il n'est pas possible d'évaluer ${ }^{23}$ l'impartialité de l'intermédiaire : les courtiers, bien organisés, bénéficient à leur avantage d'asymétries d'informations contrairement aux transporteurs et aux chargeurs qui n'ont souvent qu'une connaissance très partielle de l'offre et de la demande. Cette asymétrie leur permet «d'anticiper les variations de fret, de retenir les offres en attendant des temps meilleurs, de faire courir de faux bruits » (Le Sueur, 2005, p. 95 et p. 101) qui se répercutent sur les prix. Il en est de même des informations fournies par les deux bureaux d'information de l'Agence d'Affrètement de Charleroi quant à l'état du marché sur les trois voies de navigation qui sont en concurrence pour le transport du charbon vers la France et la Belgique. L'Agence ayant tout intérêt à développer la navigation sur les kilomètres du canal dont elle possède la concession, il est en effet difficile de penser que les informations qu'elle fournit sont toujours fiables (idem., p. 102).

Enfin, la multiplication des courtiers de capacité commerciale variable et d'origine diverse - puisqu'ils peuvent être représentants de transporteurs ou de chargeurs, mais aussi agents d'assurance, marchands ou cabaretiers - brouille l'image de la profession. Au point qu'elle ne permet plus aux chargeurs ni aux transporteurs de s'assurer de la connaissance effective que l'intermédiaire a du marché ou encore des transporteurs avec lesquels ce dernier contracte (de même que les transporteurs ne connaissent pas toujours les courtiers avec lesquels ils contractent) (ibid., pp. 94-95).

\section{L'instabilité des prix}

L'instabilité du prix est manifeste à l'époque, de manière toutefois plus marquée au $\mathrm{XIX}^{\mathrm{e}}$ siècle qu'au début du $\mathrm{XX}^{\mathrm{e}}$ (ibid., p. 99). Bien entendu, le prix tend généralement à la baisse (hausse) lorsque le marché est dominé par les chargeurs et les courtiers (bateliers), comme sur les bureaux et marchés d'affrètement (bureaux de tour). Ses variations sont liées à plusieurs facteurs (ibid., pp. 92-98 et sq) :

- les conditions météorologiques (la difficulté de remonter à vide en cas de fort vent engendre une pénurie de cales qui pousse le prix à la hausse et inversement);

- le niveau de l'activité économique (la demande de transport étant toujours une demande dérivée); la concurrence intermodale (notamment ferroviaire);

\footnotetext{
${ }^{21}$ « En théorie, le courtier fluvial est une personne qui exerce un métier réglementé par le code du commerce. Il est nommé par le gouvernement, affecté à une zone géographique précise et il lui est interdit de faire des opérations financières. Dans la réalité, cette réglementation n'est pas appliquée et le courtage est une pratique totalement libre » (Le Sueur, 2005, p. 93).

${ }^{22}$ Ces abus sont également liés à la concurrence effrénée entre courtiers, comme ce fut le cas par exemple dès lors que l'établissement de l'Agence d'Affrètement de Charleroi les rendit inutiles dans la région du Hainaut (Le Sueur, 2005, p. 103).

${ }^{23}$ Pour nous, en partie aujourd'hui, en l'absence éventuelle de sources (concernant l'Agence d'Affrètement par exemple), mais surtout à l'époque, pour les transporteurs et les chargeurs, en l'absence de réglementation (Le Sueur, 2005, p. 94 et 103).
} 
- le caractère saisonnier de l'activité de transport fluvial (liée de septembre à décembre à la campagne betteravière, aux mouvements charbonniers et à l'arrêt possible de la circulation à cause du gel, tandis que la période estivale est souvent chômée pour des raisons techniques mais aussi à cause de la sécheresse ou, au contraire, des crues);

- la manipulation des prix par les chargeurs et les courtiers résultant de leur capacité à centraliser l'information ...

Or, presqu'aucun système d'affrètement n'y remédie à l'époque, hormis dans les marchés intermédiés. L'Agence d'Affrètement de la Société du canal de la Sambre à l'Oise parvient en effet à stabiliser les variations saisonnières des prix avec à son «système de primes d'affrètement négatives ou positives »(Le Sueur, 1986, p. 43) ${ }^{24}$. Il est de même avec le système mis en place avec le tour de rôle à Dunkerque ${ }^{25}$.

Au final, les systèmes d'affrètement mis en place jusqu'en 1904 ne résolvent qu'imparfaitement les problèmes posés à l'époque à la batellerie artisanale. Des tentatives sont donc faites pour les améliorer. Pour éviter la manipulation du prix, des initiatives visent à rendre publique la disponibilité de la cale grâce à la publication par les Compagnies de la liste des bateaux passant aux écluses (Le Sueur, 2005, p. 95). Ou elles consistent à proposer de centraliser toute l'information sur la demande en un point unique où seraient également négociés tous les contrats ensuite répartis entre bateliers, comme le préconise le programme du Groupement Général de la Batellerie (Le Sueur, 1986, p. 43). Ces deux mesures peuvent résoudre le problème lié à l'asymétrie d'informations sur la demande de transport. Mais elles laissent sans réponse le problème de la fiabilité et de l'étendue de la connaissance relative à l'offre de transport que le prix est supposé transmettre également. D'où l'idée de certains courtiers, comme la maison Delegrange en 1894, d'afficher directement, de manière transparente, les prix pratiqués sur toutes les destinations sur lesquelles ils peuvent intervenir $^{26}$. Mais cette mesure ne peut être efficace que si elle est étendue à l'ensemble des acteurs... ce qui n'est évidemment pas le cas, pas plus à l'époque qu'aujourd'hui.

C'est pourquoi, malgré leurs mérites, ces différents systèmes n'empêchent nullement les conflits d'éclater, à Dunkerque et à Douai en particulier, suite à l'effondrement du prix du fret durant l'été 1904. Pour autant, l'intervention de l'Etat ne va toujours pas au-delà de sa fonction régalienne (Le Sueur, 1986, p. 44). Seule la Grande Guerre ouvrira une brève parenthèse dans cette ère libérale qui perdurera jusqu' au Front Populaire.

Pour résoudre les problèmes laissés en suspens, trois types de mesures s'avéraient en effet indispensables :

1. la réglementation de la profession de courtiers

2. la réglementation des contrats de voyage

3. la création de bureaux d'affrètement (centralisation et communication des informations (sur le prix, sur les mouvements des différents types de bateaux, sur

\footnotetext{
${ }^{24}$ Concessionnaire depuis 1832 du canal de la Sambre à l'Oise pour 99 ans, cette Société ouvre une Agence d'Affrètement en 1886 pour obtenir le quasi-monopole des voyages de charbon de charbon de Charleroi vers Paris (Le Sueur, 1986, p. 43, 2005, pp. 102-103). « Cette dernière a un triple but : informer les pénichiens sur les offres de transport et organiser des séances publiques d'affrètement; répartir les voyages selon le principe du tour de rôle ; stabiliser les cours grâce à un système de primes d'affrètement négatives ou positives » (Le Sueur, 1986, p. 43).

${ }^{25}$ Il consiste en l'établissement d'une échelle mobile de fret «pour pouvoir appliquer des règles précises de calcul des frets et éviter de trop fortes variations. Tout en respectant un plancher, les prix des voyages se calculent dorénavant selon le nombre d'unités inscrites au tour et, pour chaque bateau, en fonction de son tonnage. Ainsi, les plus petits bateaux, encore nombreux à cette époque sur ces voies, bénéficient-ils d'un fret à la tonne supérieur à celui des péniches Freycinet au chargement plus important » (Le Sueur, 2005, p. 112).

${ }^{26}$ Ce qui représentait tout de même plus de 2000 destinations pour cette maison (Le Sueur, 2005, p. 103). En 1903, le Journal de la navigation décide de publier chaque semaine une cote officielle du fret établis par plusieurs courtiers pour 46 destinations (idem., p. 104).
} 
l'offre et la demande transport), organisation des rapports entre chargeurs et transporteurs, répartition du transport entre bateliers, contrôle des conventions aux voyage).

Surtout, la généralisation et le caractère obligatoire et contraignant de la législation mise en place constituent la condition sine que non de son application effective. Or, c'est précisément sur ce point que la politique incitative échoue jusqu'en 1936.

Les mesures énoncées ci-dessus seront en effet assez rapidement proposées tant directement par les acteurs privés (dès 1907) que par le biais des incitations mises en place par l'Etat (dès 1921 sauf pour les réglementations des contrats au voyage et du courtage qui n'apparaîtront qu'en 1934) ${ }^{27}$. Mais ces tentatives de régulation du marché par les acteurs échouent jusqu'en 1936 en raison des divisions de la profession et de son incapacité (ainsi que celle de l'Etat) à rendre obligatoires et généralisées ses pratiques et ses règles. C'est pourquoi il est finalement fait appel à l'autorité de l'Etat en 1936, d'autant que la mise en ouvre des points 2 et 3 en $1914-18$ a déjà montré tout l'avantage que pouvaient en retirer les mariniers. Ce n'est pas nécessairement le cas pour les autres professions, chargeurs, courtiers et compagnies fluviales qui appuieront toujours les tendances vers la déréglementation.

\section{3/ Les coûts de transaction aujourd'hui}

Depuis 2000, le transport fluvial de marchandises en France a entièrement renoué avec l'approche libérale qui le caractérisait au tournant du siècle précédent. La réglementation instituant le Tour de rôle qui a été maintenue jusqu'en 1994, est abrogée avec un délai de six ans pour préparer les acteurs à son application. En conformité avec la directive européenne 96/75/CE du 19 novembre 1996, l'affrètement au Tour de rôle et les tarifs réglementés ont en effet été supprimés (loi n ${ }^{\circ} 2001-43$ du 16 janvier 2001). Les contrats sont désormais librement conclus et les prix, librement négociés (art.189 du Code du Domaine public fluvial de la navigation intérieure). Ce cadre institutionnel accroît des coûts de transaction qui avaient été diminués dans le cadre du Tour de rôle et en réintroduit d'autres. Si a contrario, les coûts d'organisation liés à l'existence même des bureaux d'affrètement et aux frais administratifs qu'ils entraînaient ont disparu avec la fin du Tour de rôle, la création d'un marché génère par nature de nouveaux coûts dits de transaction ${ }^{28}$ qui sont actuellement amplifiés par la crise économique. L'analyse transactionnelle repère deux catégories de coûts associés à la transaction : les coûts ex ante et les coûts ex post que nous nous proposons de reprendre ici successivement.

\subsection{Les coûts ex ante}

Les coûts de transaction ex ante correspondent aux coûts de recherche du bon partenaire et de négociation des termes du contrat. La disparition du Tour de rôle qui administrait ces aspects sur l'affrètement au voyage affecte particulièrement ces coûts.

\footnotetext{
${ }^{27}$ Notons que l'arrêté du 29 juin 1942 «réglementant les conventions d'affrètement pour la navigation intérieure et organisant les chambres syndicales des courtiers de fret »n'est aujourd'hui plus applicable. La question de la réglementation de la profession des courtiers reste à nouveau entière ( $c f$ Fischman et Lendjel, 2010).

${ }^{28}$ Notons que nous ne cherchons pas ici à comparer l'ensemble des coûts générés par les deux systèmes, avec Tour de rôle et liberté d'affrètement. Nous ne tentons que d'appréhender les coûts générés par la libre contractualisation entre les parties. Ce faisant, certains coûts de contractualisation qui existaient déjà avec le Tour de rôle (par exemple les coûts de rédaction des contrats ou encore ceux liés aux litiges entre les parties). sont au moins maintenus, D'autres (comme les coûts de coordination, ou encore ceux liés à la recherche du bon partenaire) sont créés par ce nouveau cadre institutionnel.
} 


\section{L'imparfaite information des prix}

Les coûts ex ante de transaction s'enracinent dans la nature même du transport de marchandises. Par définition, l'affrètement au voyage est un transport ponctuel et irrégulier de marchandises disponibles au point A pour être consommées au point B. Dans le transport de marchandises, la recherche d'un contrat pour l'aller implique celle d'un contrat pour le retour afin d'éviter les retours à vide. En effet, contrairement au transport de passagers où le trajet aller d'un passager implique nécessairement tôt ou tard son trajet de retour, ces deux trajets sont considérés comme deux prestations distinctes pour les transports de marchandises. Le transporteur fluvial s'apparente ainsi à une entreprise multi-produits, selon la terminologie de Baumol et al. (1977). Or, on le sait, certaines régions sont structurellement émettrices de fret, de par l'importance de leurs activités économiques, d'autres structurellement réceptrices. En raison de ces déséquilibres, le trajet de retour d'une péniche ayant transporté du fret d'une zone émettrice vers une zone réceptrice aura une probabilité non négligeable de se faire à vide. Tout transport d'un point $\mathrm{A}$ à un point $\mathrm{B}$ produit ainsi une «activité résiduelle » consistant à revenir du point $\mathrm{B}$ au point $\mathrm{A}$.

Cette caractéristique conduit à opacifier le prix de vente de la prestation de transport. Le déséquilibre des flux est en effet intégré dans le modèle économique du transporteur. Connaissant la faible probabilité d'obtenir un fret de retour, le transporteur facture son service en incluant le coût du retour. Toutefois, si un fret est à charger sur ce trajet de retour, le transporteur est disposé à le prendre quel que soit le prix proposé par le chargeur puisque son prix de revient est déjà couvert par le montant de la transaction aller ${ }^{29}$. Comme tous les autres transporteurs pratiquent cette péréquation, on peut considérer que le marché est globalement soutenable au sens de Baumol et al. (1977) ${ }^{30}$. Sauf que cet excès d'offre structurel induit une pression à la baisse du prix de transport. Car l'aller d'un transporteur peut être le retour d'un autre. Les marchés des transports de $\mathrm{A}$ à $\mathrm{B}$ et de $\mathrm{B}$ à $\mathrm{A}$ sont en effet interdépendants (National Research Council, 1978, p. 392). Le chargeur optant pour la prestation la moins coûteuse retiendra le transporteur recherchant un fret de retour qui, grâce au mécanisme de subvention croisée, proposera un «prix prédateur » en dessous de son prix de revient du seul trajet de retour (Baumol et al, 1982). Autrement dit, le transporteur fait supporter au premier chargeur une partie du coût du transport réalisé pour le deuxième chargeur (NRC, 1978, p. 392). C'est en cela que l'on a pu parler de « concurrence destructrice » (Spady et Friedlaender, 1978, p. 5), car le prix ne fournit pas à lui seul l'information suffisante pour le bon fonctionnement du marché. La remise en cause de cette concurrence destructrice entre transporteurs a historiquement toujours été une des raisons d'être centrale de la mise en place, administrée ou non, de tours de rôle. La crise actuelle exacerbant l'incertitude comportementale (Glachant et Hiroux, 2010) entre transporteurs et chargeurs ne fait qu'alimenter ce doute du prix et la crainte de comportements opportunistes des agents économiques.

Le déséquilibre des flux engendre également des coûts de transaction. En effet, la demande de transport se manifeste à un instant donné dans une région précise de façon décentralisée sans que cette information soit connue de tous. Or, l'accès à une information décentralisée est coûteuse, tant pour l'affréteur que pour le transporteur. La recherche, la transmission et le traitement de ces informations sont une source importante de coûts de transaction ex ante. Dans le transport de marchandises, ce problème est de surcroît dédoublé puisque, pour le transporteur, la recherche d'un contrat pour l'aller implique celle d'un contrat pour le retour afin d'éviter les retours à vide. Les transporteurs «atomisés » ne peuvent avoir

\footnotetext{
${ }^{29}$ L'article 209 du Code du Domaine Public Fluvial et de la Navigation intérieure prévoit pourtant de punir de $15000 €$ cette pratique.

${ }^{30}$ Ce phénomène, connu des acteurs, conduit alors parfois certains affréteurs à proposer du fret à un tarif moitié moindre dès lors qu'ils proposent un aller $e t$ un retour, selon un des artisans bateliers interrogés qui en a vécu l'expérience.
} 
une vision d'ensemble de la distribution géographique de la cale disponible à un instant donné ni de celle des besoins, d'où le risque de se retrouver dans une zone où il $\mathrm{y}$ a abondance de cales alors que celle-ci fait défaut ailleurs, et de faire un retour à vide. Ce coût de recherche d'information n'existait pas dans le système d'affrètement au Tour de rôle puisque les offres et demandes d'affrètement au voyage transitaient par les bureaux d'affrètement régionaux qui les affichaient publiquement (voir supra 2.). Au déséquilibre structurel des flux s'ajoute une organisation imparfaite de l'enchaînement des voyages résultant de l'importance de ces coûts. Cela renforce en outre le coûteux phénomène de concurrence destructrice, mentionné précédemment.

\section{L'intermédiation du courtier, la réponse du secteur fluvial aux coûts de transaction}

L'existence de courtiers constitue la réponse produite par le secteur fluvial pour réduire les coûts importants de transaction résultant des coûts de production de l'information et de rencontre des acteurs. Si l'existence de courtiers a grandement amélioré la quantité d'informations produites et leur qualité, leur dispersion engendre néanmoins toujours des coûts qui restent élevés. L'absence de centralisation des informations relatives à la cale du côté des transporteurs pose en effet un véritable problème pour les affréteurs qui ont également vu leur temps et frais de communication considérablement alourdis ${ }^{31}$. C'est toutefois moins le cas pour les chargeurs car ils travaillent généralement avec quelques courtiers ou commissionnaires spécialisés dans le transport de leur output et/ou sur la zone dans laquelle celui-ci s'effectue - et reportent donc sur ces derniers cette opération de recherche de cale adaptée disponible. En revanche, les transporteurs doivent sans cesse appeler les courtiers et répondre à leurs appels pour les informer de la disponibilité de leur cale et trouver du fret. Ils échangent aussi par internet. Ces appels à la charge des transporteurs (courtiers) sont d'autant plus nombreux et coûteux que le fret est rare (abondant) bien entendu, mais aussi que les courtiers sont nombreux. En l'absence de courtiers, ce coût serait probablement décuplé (si l'on inclut le temps à y consacrer). Les transporteurs sont alors face à plusieurs interlocuteurs, même si ils entretiennent généralement des relations privilégiées avec un petit nombre d'entre eux $(3 \mathrm{ou} 4)^{32}$. Leur difficulté, tout comme celle des courtiers, est de fournir et d'obtenir en permanence et à moindre coût une information la plus complète possible sur l'état du marché pour faciliter leur mise en rapport avec les chargeurs. La facture téléphonique, indépendamment du temps consacré à cette occupation, peut représenter entre 1 et $1,5 \%$ du chiffre d'affaires d'un batelier ${ }^{33}$. Or, plus ce coût est élevé en raison de l'affaiblissement de la demande de transport, plus les transporteurs sont incités à accepter les premiers contrats qui se présentent, fût-ce à un taux de fret peu élevé.

\section{Les coûts d'intermédiation, mesure approximative des coûts de transaction ex ante}

Le service rendu par le courtier a bien entendu un prix, celui de l'intermédiation. Il correspond à une commission d'affrètement, proportionnelle au taux de fret, payée par le transporteur au courtier. Le niveau de cette commission constitue une mesure directe - même approximative - des coûts de transaction résultant du nouvel environnement institutionnel. Ils

\footnotetext{
${ }^{31}$ Le temps passé à cette recherche a ainsi été « multiplié par quatre » depuis la disparition de Cale infos service, selon M. Kerrotret de Logistra.

${ }^{32}$ Les relations de confiance développées entre les parties interviennent ici pour limiter les risques de comportement opportunistes et les doutes sur le prix.

${ }^{33}$ Ces calculs sont effectués à partir des Livrets 2007 et 2008 mis à notre disposition par un des artisans batelier disposant d'une péniche Freycinet.
} 
constituent un transfert de coûts auparavant supportés (sous forme de coûts d'organisation) par l'ONN pour faire fonctionner le Tour de rôle et les bureaux d'affrètement.

Cette commission est souvent de 5\%. Ce taux peut être pratiqué tant pour l'import/export que sur les transports intérieurs. Il varie assez peu selon les affréteurs ou encore le gabarit du bateau ${ }^{34}$. Toutefois, il peut atteindre jusqu'à $7,5 \%$ voire $8,5 \%$ sur les marchés intérieurs ${ }^{35}$.

De plus, les affréteurs exigent des transporteurs des frais de dossier pour chaque contrat, en sus de leur commission. Or ces frais ne sont pas mentionnés dans les contrats. Abusifs, ils sont en quelque sorte d'usage ${ }^{36}$. Les transporteurs parviennent toutefois à les négocier lorsque ces contrats sont réguliers. Dans ce cas, ils peuvent par exemple être amenés à ne payer que quelques frais de dossiers sur l'ensemble des contrats réalisés ${ }^{37}$.

Enfin, les affréteurs exigent encore souvent une prime de Ducroire aux transporteurs. Autrefois destinée à alimenter une caisse permettant d'indemniser les transporteurs en cas de défaillance du client chargeur, cette prime ne devrait plus exister aujourd'hui. En effet, avant d'effectuer un fret, les transporteurs devraient avoir obtenu la preuve qu'une garantie a bien été prise pour couvrir désormais ce risque. Cette prime représente entre $0,5 \%$ et $2 \%$ du prix du fret transporté.

$\mathrm{Au}$ total, le coût d'intermédiation représente près d'un dixième du CA $(9,3 \%$ en 2007 et $8,1 \%$ en 2008 ) $^{38}$ d'un artisan batelier équipé d'une péniche Freycinet (soit $75 \%$ de la batellerie artisanale) si l'on ajoute ses frais de déplacement (donc de représentation), ses frais téléphoniques et ses frais de dossier. On peut supposer, au vu de la récurrence de ces transactions ${ }^{39}$, que ce montant est assez représentatif du secteur, sans toutefois pouvoir le fonder sur la base d'un échantillon réellement représentatif.

A cette commission d'affrètement versée par le transporteur au courtier s'ajoute parfois une marge prise par le courtier dans le contrat de commission que lui confie le chargeur. En effet, ce dernier est généralement à l'origine de la transaction. Il mandate le courtier pour qu'il organise son transport fluvial en affrétant un transporteur. Le courtier agit ainsi en tant que commissionnaire de transport pour le compte du chargeur et en tant que courtier (représentant donc les intérêts du transporteur) auprès du transporteur. Le courtier profite ainsi parfois de cette confusion des rôles de courtier et de commissionnaire pour surfacturer son intermédiation.

Cette confusion est rendue possible par le fait que 1/ les courtiers sont souvent également commissionnaires de transport, 2/ les contrats ne mentionnent pas à quel titre l'affréteur intervient, 3/ le prix négocié avec le chargeur n'est pas forcément connu du transporteur. Dès lors, le courtier a le pouvoir, pourtant illégal, d'imposer au transporteur le niveau de sa commission d'affrètement une fois qu'il est mandaté et rémunéré à cette fin par le chargeur pour affréter un transporteur fluvial. Relevons que cette confusion n'est d'ailleurs pas nouvelle. En 1983, le rapport Grégoire relevait déjà cette confusion des rôles entre le courtage et la commission de transport. «Un arrêté ministériel du 29 juin 1942 donne du courtage une définition tellement étendue qu'il confine aux fonctions des commissionnaires de transport, sans atteindre toutefois le niveau des responsabilités assumées par ces

\footnotetext{
${ }^{34}$ Sur la dizaine d'affréteurs (courtiers ou commissionnaires de transport) avec lequel travaille l'artisan batelier qui nous a remis ses carnets, la SCAT représente environ le tiers de ses affrètements. Notons que ce taux diffère même au sein de la SCAT (entre la SCAT Compiègne et la SCAT Reims).

35 selon les informations communiquées par les quatre artisans bateliers que nous avons interrogés.

${ }^{36}$ Ces frais s'élèvent entre $5 €$ et $22,87 €$ par contrat, soit en moyenne $0,23 \%$ du CA.

${ }^{37}$ De l'ordre de 3 frais de dossiers sur 8 contrats, selon un des bateliers que nous avons interrogé.

${ }^{38}$ Ces calculs sont effectués à partir des Livrets 2007 et 2008 mis à notre disposition par le batelier. L'absence de toute référence à l'intermédiation de «Cale Info Service» en 2008 suite à sa disparition explique la diminution des frais d'intermédiation de 1 point.

${ }^{39} 61$ transactions sur 2 ans pour l'artisan qui a mis à notre disposition ses livrets 2007 et 2008.
} 
intermédiaires »(Grégoire, 1983, p. 71). Reste qu'avec la libre contractualisation entre les parties, cette confusion peut se traduire par des abus parfois constatés par les transporteurs lorsqu'ils parviennent à savoir pour quels chargeurs ils opèrent et, surtout, à quel prix les transports leur ont été facturés ${ }^{40}$.

Ainsi, la somme de ces coûts ex ante de transaction - hors temps passé par les acteurs représente probablement plus de $10 \%$ du montant de la transaction. Par comparaison, l'Office National de la Navigation évaluait, dans une étude sur les prix de revient en 1981 (O. N. N., 1982), à 6,25\% (soit 4 points de moins qu'en 2008) le coût moyen d'intermédiation (sur un échantillon de 82 exploitants) ${ }^{41}$. Les bureaux d'affrètements produisaient donc du temps du Tour de rôle un service d'intermédiation près de $30 \%$ moins cher qu'aujourd'hui. Ce renchérissement s'explique probablement pour deux raisons : 1/ la diminution d'économie d'échelles résultant d'un nombre accru d'intermédiaires pour un nombre décroissant de transporteurs et de chargeurs ; 2/ les acteurs privés, contrairement à l'organisme public, tarifient leur intermédiation en intégrant un taux de marge.

Le recours au marché a donc incontestablement accru les coûts ex ante de transaction (annexe, tableau 1).

\subsection{Les coûts ex post de transaction}

Les coûts ex post repérés sont liés aux risques de comportements opportunistes des agents lors de la réalisation effective de la transaction. On l'a vu, ils tiennent, d'une part, à la rédaction non systématique des contrats et, d'autre part, aux délais de paiement des surestaries.

\section{Incertitude comportementale en l'absence de contrats}

$\mathrm{Si}$ les contrats sont aujourd'hui réglementés, ils ne sont plus contrôlés systématiquement depuis la fin du Tour de rôle. Or, l'absence de contrôle des conventions au voyage ouvre la porte à tous les abus de la part des affréteurs. La question ici est celle des coûts ex post générés par le non-respect de la réglementation.

De fait, les bateliers ne parviennent pas à obtenir systématiquement des contrats écrits dans le respect des textes. En particulier, par souci de réactivité, ils peuvent convenir par écrit (par courriel) ou oralement (par téléphone) avec l'affréteur de transporter un fret, sans toutefois recevoir de document stipulant les dates de chargement et de livraison, la quantité à charger, le prix etc. En ce cas, les transporteurs sont à la merci de toutes les modifications et abus qui peuvent en résulter. Par exemple, un taux de fret convenu oralement au téléphone peut s'avérer inférieur lorsque le transporteur se présente à quai pour charger la marchandise. La perte de temps pour se rendre sur place et le refus d'autres propositions éventuelles de contrat peuvent inciter le transporteur à accepter ce coup de force opportuniste. L'existence de contrats types dit «supplétifs » ne permet pas en effet de remédier à cette situation parce qu'ils ne peuvent fournir de façon générale des éléments sur le montant et la durée de la transaction ou sur les conditions de la manutention. De graves dysfonctionnements dans le déroulement même de la prestation et dans la répartition des frais liés à ces dysfonctionnements peuvent ainsi se produire à la suite de comportements opportunistes.

Pour les éviter, nous avons déjà noté que les transporteurs continuent de s'appuyer sur des relations de confiance qu'ils ont établies avec quelques courtiers pour effectuer la plupart

\footnotetext{
${ }^{40}$ Comme en ont fait plusieurs fois l'expérience les bateliers que nous avons interrogés. Il convient néanmoins de noter que cette information n'est pas affichée sur la place publique, les contrats étant conclus de gré à gré dans le secret des affaires. Le marché du transport est de ce point de vue d'une très grande opacité, en particulier pour les transporteurs.

${ }^{41}$ Ces coûts comprenaient les commissions d'affrètement et les frais d'utilisation des bureaux d'affrètement.
} 
de leurs transports. Mais ils ne garantissent pas à eux seuls un fonctionnement optimal du marché. Les abus ne font qu'accroître les coûts supportés par les transporteurs en position de faiblesse - comme ceux que nous avons précédemment mentionnés plus haut au sujet des coûts d'intermédiation (frais de dossier et prime de Ducroire).

\section{Incertitude comportementale dans la réalisation du contrat}

Dernière difficulté transactionnelle posée aux transporteurs : le délai de paiement des surestaries. Correspondant aux indemnités dues aux bateliers en cas de retard, c'est-à-dire en cas de dépassement du délai de planche ${ }^{42}$, les surestaries ne sont pas toujours payées dans les temps aux bateliers. Ceux-ci doivent alors les réclamer, ce qui est coûteux pour eux mais également pour le courtier qui les a affrétés et qui doit se retourner vers l'expéditeur ou plus souvent le destinataire qui est l'auteur de ces délais ${ }^{43}$. Or, ce coût est d'autant plus lourd à payer que les montants de surestaries indiqués par VNF sont insuffisants pour indemniser au coût réel les frais supportés par les bateliers qui n'ont pu charger et/ou livrer à temps.

En effet, le montant des surestaries est forfaitaire et ne correspond jamais au dommage effectivement subi par le transporteur. En particulier, il introduit un aléa d'exploitation qui empêche le transporteur de proposer ses services à un tiers à une date fixée à l'avance. Il subit ainsi la perte la plus importante résultant de l'immobilisation de son bateau. Comme ce dernier constitue une sorte d'entrepôt flottant pour le destinataire, les surestaries versées par ce dernier - si le délai est de son fait - sont certes probablement supérieurs aux coût de stockage correspondant à terre, mais peu pénalisant pour le destinataire. Le transporteur, en revanche, peut perdre d'importantes opportunités de transaction. Le taux de surestaries fixé par décret (mais non obligatoire) à 143,61€/jour pour une péniche de type Freycinet est d'un montant quatre fois inférieur au CA moyen/jour d'affrètement de l'artisan batelier interviewé. A l'inverse, en l'absence d'opportunité d'affrètement, le transporteur bénéficie d'une rémunération supplémentaire qu'il aurait perdue si les délais avaient été respectés par le chargeur. Si un dispositif symétrique existe pour les dépassements de délai de parcours par les bateliers (d'un montant journalier de 114,95€), leur ponctualité légendaire (provenant de la forte incitation monétaire à respecter les délais) le rend pratiquement virtuel.

Les surestaries correspondent ainsi à une police d'assurance souscrites par deux agents permettant de couvrir la réalisation d'un risque entre ces deux agents. Elle évite les coûts de renégociation ex post entre les deux parties en cas de réalisation de ce risque. Pour autant, on peut s'interroger sur le bienfondé de ce dispositif car cette modalité de partage de la quasirente ne permet pas de valoriser à sa juste mesure cette externalité (Pirrong 1993, p. 942). Le questionnement de ce dispositif est important si l'on veut augmenter les gains de productivité de ce secteur. De fait, ces délais peuvent avoir des effets en cascade sur les voyages à venir du transporteur.

Ainsi, il existe bien d'importants coûts de transaction ex ante et ex post générés par l'organisation actuelle du marché du transport fluvial telle qu'elle est définie par la réglementation depuis 2000. L'analyse de ces coûts donne un éclairage sur le comportement des acteurs, en particulier au fait qu'ils adoptent des comportements pro-cycliques, préjudiciables, en période de crise comme aujourd'hui, à l'ensemble de la profession opérant dans le transport fluvial de marchandises. C'est pourquoi il convient de réfléchir à des possibles corrections. C'est en ce sens que nous avançons à présent quelques pistes de recommandations.

\footnotetext{
${ }^{42}$ voir définitions plus haut note 18 .

43 «Comme il y a souvent des délais supplémentaires non prévus, il y a une vraie bagarre sur les surrestaries » souligne P.-Y. Kerrotret de Logistra dans un interview.
} 


\section{Recommandations}

L'importance des coûts de transaction recensés plus haut pourrait être réduite si certaines mesures étaient adoptées. Nous en proposons deux, en lien avec l'approche transactionnelle que nous avons adoptée dans ce travail ${ }^{44}$. Tout d'abord, la mise en place de bourse d'affrètement en ligne ouverte à tous les acteurs mais essentiellement alimentée par les transporteurs. Ensuite, la création d'un référentiel de prix de revient et d'outils d'aide au calcul de prix de revient individuel par un organisme indépendant.

\subsection{La bourse de fret}

La première proposition est celle d'une bourse de fret en ligne ouverte à tous les acteurs mais essentiellement alimentée par les transporteurs. Cette idée n'est pas originale. Elle était ainsi déjà mentionnée par Fabart et al (1986, pp. 80-84) pour améliorer le fonctionnement du Tour de rôle. Comme nous l'avons mentionné dans notre deuxième partie (supra 2), le principe même des bourses d'affrètement existe d'ailleurs depuis le $\mathrm{XX}^{\mathrm{e}}$ siècle. Jusqu'en 2009, date de sa disparition, Cale Info Service (CIS) proposait un système de ce type. Aujourd'hui il existe à notre connaissance, en France, une bourse en ligne proposée par Entreprendre pour le Fluvial (EPF). Elle est peu utilisée par les bateliers en partie parce qu'EPF est un organisme relativement nouveau dans le secteur. Enfin, il existe une bourse en ligne mise en place depuis 2005 par des artisans indépendants au niveau européen sur le portail du transport fluvial (http://batellerie.org/texts/a-propos-de). Soixante-deux bateliers (pour environ 700 artisans bateliers en France!) y sont inscrits. Ils cotisent pour assurer le fonctionnement du site (216 euros par an en 2010). L'objet de celui-ci est de fournir 1/ une visibilité sur la cale disponible et ses caractéristiques et $2 /$ le contact des bateliers. Ainsi, les affréteurs peuvent trouver une offre de cale répondant à leurs besoins qu'ils négocient librement avec le transporteur de leur choix. Par ailleurs, il est possible pour les transporteurs d'y proposer des demandes de fret auxquelles ils ne peuvent (ou ne veulent) répondre, au bénéfice des autres membres du site. Les affréteurs partenaires peuvent faire de même. Nous proposons que les transporteurs utilisent également le site de la bourse pour transmettre les contrats qu'ils signent avec les affréteurs.

Sa limite est la difficulté technique qu'ont les bateliers à accéder au réseau internet en raison de leur mobilité. L'obstacle financier est également à prendre en compte. La difficulté des artisans bateliers, professionnels indépendants, à voir l'intérêt d'un fonctionnement en réseau est un dernier problème.

Cet intérêt est pourtant multiple. La bourse en ligne peut bien entendu fournir une cale de réserve. Mais cette fourniture ne peut être utile à tous les acteurs qu'en cas de forte demande de transport, nullement en cas inverse, comme en témoigne la fermeture de Cale Info Service en 2009. En revanche, dès lors que cette bourse est utilisée par une grande majorité d'artisans, les effets réseaux de la bourse en ligne peuvent être importants.

Outre le fait que la bourse donne à voir la profession référencée sur le site, l'autre intérêt de ce système, mentionné sur la page d'accueil du site, est de réduire les coûts de transaction ex ante (en particulier les coûts d'information) en améliorant la disponibilité de l'information et son accès grâce à sa centralisation. Par la transmission des contrats via la bourse en ligne, les bateliers pourraient également réduire les coûts d'intermédiation et les coûts ex post. Outre de garantir le taux de fret convenu oralement, ils s'assureraient en effet par ce biais que les contrats soient toujours écrits et conformes à la législation en vigueur. De

\footnotetext{
44 A ce titre, nous ne reprendrons pas une proposition souvent faite (Fabart et al, 1986 ; Damien, 2009 ; Eurotrans, 2009) de regroupement des artisans en coopératives par exemple, leur permettant d'agir sur leur fonction de coûts de production.
} 
ce dernier point de vue, les affréteurs seraient notamment tenus, du fait de ce contrôle, de mentionner à quel titre ils interviennent (en tant que courtier, commissionnaire de transport ou chargeur). La bourse permet également à la profession de bateliers d'être plus réactive, par rapport aux demandes des chargeurs. Car elle permet à des bateliers de satisfaire ces demandes, si d'autres artisans, à qui cette demande était initialement adressée, ne peuvent isolément y répondre ${ }^{45}$. La bourse facilite, de surcroît, l'insertion du transport fluvial de marchandises dans la chaîne logistique. Elle constitue aussi un outil de dialogue au sein de la profession $^{46}$.

Pour toutes ces raisons, le site en ligne des artisans bateliers améliore l'organisation de l'enchaînement des voyages, limite le déséquilibre structurel des flux et, ainsi, le coûteux phénomène de concurrence destructrice. Encore faut-il que le site soit alimenté régulièrement par une grande partie des bateliers. Sinon, l'information reste incomplète. En revanche, plus il y a de bateliers, plus il est intéressant pour eux d'y participer - car, en raison des coûts que cela engendrerait, plus faible serait la probabilité que les affréteurs fassent appel à un transporteur non inscrit sur le site. Pour atteindre un tel objectif dans le transport fluvial de marchandises, il faudrait donc que les bateliers acceptent d'agir collectivement à travers cette bourse en ligne - ce qui n'est pas simple pour une profession très attachée à son indépendance, où chacun se pense non seulement comme un entrepreneur individuel (l'artisan, le gérant d'une TPE) mais aussi comme une profession libérale (libre de choisir le lieu et la manière d'exercer son métier). Pourtant, dans le cadre d'un marché librement organisé, il semble que c'est aussi à la profession de se donner les moyens de son développement en se dotant des outils et en adoptant les comportements qui l'aideraient à diminuer ses coûts et à faciliter les transactions dans le secteur.

\subsection{La création de référentiels de prix de revient et d'un outil d'aide au calcul du coût de revient individuel}

A la suite de la disparition de la gestion administrée de l'affrètement au voyage, les artisans bateliers ne connaissent pas bien leur prix de revient. Ils ont du mal à évaluer leurs coûts de fonctionnement et, surtout, leurs coûts fixes. Par exemple, le batelier ne provisionne pas le coût de remplacement du bateau ${ }^{47}$. Les bateliers indépendants se retrouvent dans une situation analogue à celle qu'ont connue les professionnels du transport routier à la fin des années 80. Ils peuvent donc être amenés à proposer des prix trop faibles, ie, engendrant un phénomène de concurrence destructrice. De plus, sans connaissance du coût de revient moyen de la profession, il est difficile pour les acteurs de déterminer le prix en dessous duquel les offreurs travailleraient à perte.

Deux arguments plaident ainsi en faveur de la mise en place de référentiels de prix de revient et d'outils d'aide personnalisée au calcul de ces prix dans le secteur du transport fluvial. 1/ Ils permettent de diminuer les coûts de transactions; 2/ ils s'inscriraient logiquement dans le dispositif régulateur mis en place par l'Etat.

\section{Référentiel de prix de revient et coûts de transaction}

Comme évoqué plus haut, le problème de lisibilité des prix et des coûts est particulièrement aigu, notamment en niveau, dans le cadre des affrètements au voyage ${ }^{48}$. Il

\footnotetext{
${ }^{45}$ Pour autant, ce n'est pas dire que la bourse supprimerait le rôle du courtier, bien au contraire. Celui-ci réduit également ses coûts et améliore sa connaissance des acteurs.

${ }^{46}$ Ces trois derniers points avaient déjà été mentionnés dans Fabart et al , 1986, pp. 80-84.

${ }^{47}$ C'est ce qui apparaît dans les carnets de l'artisan batelier interviewé.

${ }^{48}$ Dans le cadre des affrètements au tonnage ou à temps, le principal problème concerne les dérives de coûts dans le temps. Des indices de coût, permettant de mesurer cette dérive, constituent une réponse institutionnelle adéquate.
} 
intervient au moment même de la négociation du contrat, puisque le prix proposé peut être extrêmement variable.

Lors d'une prestation unique, le coût de transaction doit être le plus faible possible pour les deux parties. Le temps de négociation doit être réduit au maximum, le temps d'acquisition des informations pertinentes également, etc. Pour cela, une solution simple est de faire appel aux bourses de fret comme on l'a vu précédemment, mais ce n'est évidemment pas la seule. Sur une bourse de fret, le prix n'apparaît généralement pas en ligne puisqu'il est négocié directement entre les deux parties. Dans cette procédure, les coûts de transaction sont assez faibles, mais les informations relatives au contenu des prestations sont inexistantes, générant des risques pour les deux parties contractantes.

Des référentiels de prix de revient fournissent une information collective - établie par enquête auprès d'un échantillon représentatif de transporteurs - dans une négociation particulière entre deux individus. Quel peut-être l'intérêt d'une telle information dans le cadre d'une rencontre bilatérale, compte tenu des spécificités de la prestation négociée et des conditions particulières de son exécution?

- Pour le donneur d'ordre, l'intérêt est double : $1 /$ un référentiel de prix de revient fournit des informations sur les coûts à supporter pour une prestation moyenne, dans des conditions d'exploitation moyenne. Plus la prestation à réaliser comporte une dimension spécifique, plus le risque augmente que le transporteur ayant accepté ce fret en spot ne respecte pas cette spécificité. En effet, plus le prix est faible, plus le risque augmente que la prestation fournie soit de mauvaise qualité ou qu'il travaille à perte. Autrement dit, la seule information prix ne permet plus de sélectionner le bon partenaire. Un référentiel de coût fournit une information complémentaire au chargeur lui permettant de mieux apprécier le risque. $2 /$ un référentiel de coûts permet d'évaluer la proposition individuelle du transporteur et de la situer par rapport à cette moyenne. Si la proposition tarifaire est significativement en dessous du prix, il peut demander des détails sur les conditions de la prestation; si la proposition est significativement supérieure, il est en mesure de demander des explications au transporteur, afin de négocier au mieux le prix.

- Côté transporteur, un référentiel de prix de revient permet : $1 /$ de situer le coût de sa prestation par rapport à la concurrence; $2 /$ de justifier ses prix dans le cadre d'une négociation à partir d'informations extérieures, pour ne pas dire "objectives", aux deux parties. 3/ d'indiquer au chargeur une sorte de plancher en dessous duquel il encourt le risque du délit de prix abusivement bas (art 209 du Code du Domaine Public Fluvial et de la Navigation intérieure). Un prix supérieur au niveau du référentiel de coût permet donc en quelque sorte de "garantir" au donneur d'ordre la licéité de la prestation proposée.

Que se produit-il en l'absence de référentiels de prix de revient ? Les deux parties doivent se procurer les informations nécessaires à la transaction. Au regard des coûts évoqués plus haut (délais et coûts d'acquisition de l'information sur la prestation, délais de négociation, recherche d'autres prestataires, prise en compte des contraintes réglementaires, etc.), le processus de négociation est plus long et plus coûteux pour les deux parties. En l'absence de points de repère externes, le temps nécessaire pour que les deux parties s'accordent sur le prix et sur le contenu de la prestation s'allonge. Des référentiels de prix de revient fournissent gratuitement (ou à moindre coût) et de manière synthétique des informations qui autrement sont coûteuses et difficiles à synthétiser pour les parties en présence.

En somme, ces outils constituent des éléments de connaissance commune, partagés par tous, qui permettent de stabiliser les représentations et les anticipations des entrepreneurs (transporteurs et chargeurs) et de diminuer leurs coûts de transaction. Rappelons à ce propos que, dans la programmation des flux de transports, il importe aux transporteurs comme aux 
chargeurs de pouvoir budgéter à l'avance leurs coûts (pour les chargeurs) et leurs revenus (pour les transporteurs, puisque le prix est un élément décisif de la justification d'un investissement). Chacun insère la prestation de transport dans une chaîne budgétaire qui lui est propre.

\section{Le rôle de référentiels de prix de revient dans le dispositif de réglementation et de régulation du secteur}

Il convient ici également de rappeler un élément de nature réglementaire. L'article 209 du chapitre V du Code du Domaine Public Fluvial et de la Navigation intérieure

«puni d'une amende de 15000 euros le fait pour tout prestataire de transport public de marchandises par voie navigable, auxiliaire de transport ou loueur de bateaux de marchandises avec équipage, d'offrir ou de pratiquer un prix inférieur au coût de la prestation qui ne permet pas de couvrir les charges entraînées par les obligations légales et réglementaires, notamment en matière sociale et de sécurité, ainsi que les charges de carburant et d'entretien, les amortissements ou les loyers des bateaux, les frais de péage, les frais de documents de transport, les timbres fiscaux et, pour les entreprises unipersonnelles, la rémunération du chef d'entreprise ».

Pour être opératoire, le principal organisme de contrôle (la DGCCRF) et les transporteurs tiers se portant partie civile doivent pouvoir s'appuyer sur une connaissance des niveaux moyens de coûts pour une prestation normale. De surcroît, un transporteur évincé ne peut s'appuyer sur ses propres conditions d'exploitation pour mettre en cause celles d'un concurrent. Ces acteurs doivent donc disposer de référentiels de coûts élaborés par un organisme indépendant afin d'étayer les présomptions. Les référentiels de prix de revient sont donc des supports institutionnels permettant à tous de disposer des informations nécessaires au respect de la loi.

Ainsi, indépendamment de toute considération sur l'utilité de référentiels de prix de revient pour les parties contractantes, les pouvoirs publics se doivent d'élaborer de tels repères pour que son dispositif de régulation du secteur soit tout simplement cohérent ${ }^{49}$.

On notera que ces deux mesures peuvent exister séparément. Elles gagneraient néanmoins à être proposées simultanément en raison de leur complémentarité et des effets de réciprocité qu'elles génèrent l'une par rapport à l'autre. Si toutefois ce n'était pas le cas, la plus essentielle à réaliser est la création du référentiel de prix de revient et des outils de calcul du prix de revient individuel par un organisme indépendant. En effet, nous avons vu que cette mesure constitue en quelque sorte le socle sur lequel repose l'efficacité de la bourse en ligne. De plus, dépendant de la volonté des pouvoirs publics, elle ne serait pas directement soumise au comportement des acteurs (contrairement à la première mesure). En revanche, elle leur donnerait un signal fort quant à l'importance accordée au transport fluvial en France aujourd'hui.

\section{Conclusion}

Avec la remise en cause du Tour de rôle, la libéralisation du transport fluvial de marchandises a réintroduit des coûts de transaction que les systèmes d'affrètement expérimentés à la période libérale au tournant du $20^{\text {ème }}$ siècle n'étaient pas parvenus à éliminer (opacité du prix de vente et prix prédateurs, coûts de recherche d'information élevés, coûts d'accès importants à l'intermédiation, coût d'intermédiation élevés, facturation des temps d'attente, etc.). Or ces coûts handicapent lourdement la profession des artisans bateliers, pourtant dominants dans le secteur. Pour y remédier et inscrire le secteur dans le sens du Grenelle de l'environnement, il

\footnotetext{
${ }^{49} \mathrm{La}$ demande d'un tel dispositif était au cœur des revendications du mouvement social d'avril 2010. Il figure en bonne place dans le protocole de sortie de crise signé à Paris le 5 mai 2010 entre la CNBA, le CAF, La Glissoire, plusieurs courtiers et commissionnaires, en présence des représentants de l'Etat.
} 
faudrait que les artisans participent largement à une bourse en ligne et que les pouvoirs publics créent un dispositif d'observation des prix de revient et d'aide personnalisée à leur calcul. Toutefois, il est évident que «ou le système judiciaire fait son travail; ou le système de marché ne pourra plus être rétabli $»^{50}$.

\footnotetext{
${ }^{50}$ Comme l'écrit James K. Galbraith, in Le Monde du 21 mai 2010, p. 18, à propos des marchés financiers.
} 


\section{Bibliographie}

Allam D. et Lendjel E. (2009), "La franchise : une gouvernance pour les entreprises de transport routier de marchandises?", in Baudry B. et Dubrion B. (eds.) Analyses et transformations de la firme : approches pluridisciplinaires, Paris, La Découverte, 2009

Arrunada B., Gonzales-Diaz M., Fernandez A. (1998), «Contractual and regulatory explanations of quasi-integration in the trucking industry », Working Paper presented at Atom, Université de Paris1 Panthéon-Sorbonne.

Baumol W. J., Bailey E. E., Willig R. D. (1977), "Weak invisible hand theorems on the sustainability of multiproduct natural monopoly", The American Economic Review, Vol. 67, n 3, 350-360.

Baumol W. J., Bailey E. E., Willig R. D. (1982), Contestable Markets and the Theory of Industry Structure, San Diego, Harcourt Brace Jovanovich, 1982.

Cap à l'amont, n 37, 6 mai 2010.

Damien M.-M. (2009), Transport et logistiques fluviaux, Paris Dunod, 2009.

Eurotrans (2009), Etude des perspectives à moyen terme de l'activité et de la flotte fluviale, Mission auprès de l'Association Entreprendre pour le fluvial, Paris, 95 p.

Fabart A., Huber J., Jubelin L., Postel J.-Y. (1986), Viabilité économique de l'artisanat batelier et insertion dans les chaînes de transport, Etudes et recherches économiques industrielles, Ministère des Transports, de l'Urbanisme et du Logement, Boulogne, 1986, 158 p.

Finon, D. et Perez, Y. (2007), "The social efficiency of instruments of promotion of renewable energies: A transaction-cost perspective ", Ecological Economics, Vol. 62, Issue 1, 77-92

Fischman, M. (1998), "Le concept quesnayen d'ordre naturel", Cahiers d'Economie Politique, ${ }^{\circ}{ }^{\circ} 32$, L'Harmattan, pp. 67-93.

Fischman M.et Lendjel E. (2010), «Efficience du marché et contrats types: une analyse transactionnelle du contrat type d'affrètement au voyage dans le transport fluvial de fret », Les Cahiers Scientifiques des Transports, $\mathrm{n}^{\circ}$ 59/2011, soumis pour publication.

Friedlaender (1978), «Hedonic cost and economies of scale in the trucking industry », in The National Research Council (1978), 33-56

Glachant J.-M. et Hiroux C. (2010), «L'énergie éolienne : comment insérer une production inflexible dans une chaîne déintégrée de «juste à temps »? », Reflexive Governance in the Public Interest, Sixth framework programme, Working http://refgov.cpdr.ucl.ac.be/?go=publications \&dc=3950c6cba81da465046f 214377fd39a 1bf1b884e

Paper,

Grégoire R. (1983), Schéma de développement du transport fluvial et schéma directeur des voies navigables ; rapport de la commission présidée par Mr Grégoire, Ministère des Transports, Ministère du Plan et de l'Aménagement du Territoire, Paris, 92 p.

Gerritsen D. (1983), Du Fleuve à la terre : la reconversion obligée des travailleurs du transport fluvial ... Mobilité professionnelle - mobilité sociale ?, Monographie, Ministère des transports, Paris, mai 1989.

Lamy Transport (2009), Route, transport intérieur et international, T.1, édition 2009, Wolters Kluwer France, Rueil-Malmaison.

Le Sueur B. (1982), Les pénichiens, mariniers du Nord - 1850)1950 - Essai d'histoire sociale, Thèse de $3^{\text {ème }}$ cycle sous la direction de Joseph Goy, Université Paris VII, mai 1982.

Le Sueur B. (1986), «L'Etat et l'adaptation du transport fluvial aux mutations économiques », in Pierre Tripier, Travailler dans le transport, Paris, L'Harmattan, coll. Logiques sociales, 1986, pp. 3756. 
Le Sueur B. (1993), «Les adaptations réglementaires et sociales de la batellerie industrielle face aux évolutions de son marché », in Hamelin P., Ribeill G. et Vauclaire C., Transport 93, Professions en devenir, Paris, Presse des ponts et Chaussée, pp. 65-86.

Le Sueur B. (2004), Histoire et mémoire de la batellerie artisanale, tome 1, Douarnenez, Editions du Chasse-Marée, Glénat.

Le Sueur B. (2005), Histoire et mémoire de la batellerie artisanale, tome 2, Douarnenez, Editions du Chasse-Marée, Glénat.

Ménard C. (1997), L'économie des organisations, Paris, La Découverte, collection Repères.

Masten, S.E., Saussier, S. (2002), "Econometrics of contracts: an assessment of developments in the empircal literature on contracting", in Brousseau, E et Glachant, J.-M. (Eds.), The Economics of Contract: Theories and Application. Cambridge, Cambridge University Press, pp. 273-290.

National Research Council (1978), Motor Carrier Economic Regulation; Proceeding of a workshop conducted by Committee on Transportation with the Transportation Center at Northwestern University, US Department of Transportation, National Academy of Science, Washington, D. C. 1978.

Neiertz N. (1999), La coordination des transports en France : de 1918 à nos jours, Comité pour l’Histoire économique et financière de la France, Paris, 1999.

Office National de la Navigation (1982), Enquête sur les prix de revient du transport fluvial (conditions économiques de 1981), $41 \mathrm{p}$.

Pirrong, S.-C. (1993), "Contracting practices in bulk shipping markets: A transactions cost explanation”, Journal of Law \& Economics, Vol. 36, n², 937-976.

Pugin M (1982), L'histoire $d u$ Canal Saint-Quentin, MFSHAA, t. XXVII, 43-60, http://www.histoireaisne.fr/memoires_numerises/chapitres/tome_27/Tome_027_page_043.pdf

Spady R. H. et Friedlaender A. (1978), "Hedonic cost functions for the regulated trucking industry », The Bell Journal of Economics, Vol. 9, n 1, 159-179.

Williamson, O. (1985), The economic institutions of capitalism, New York, The Free Press, 1985.

Williamson O. (1989), "Transaction cost economics», in: R. Schmalensee \& R. Willig (ed.), Handbook of Industrial Organization, Elsevier, Vol. 1, Chapter 3, 135-182.

Williamson O. (1993), "Calculativeness, trust and economic organization », Journal of Law and Economics, Vol. 36, No. 1, Part 2, 453-486.

Williamson, O. (1996), The mechanisms of governance, Oxford, Oxford University Press, 1996. 


\section{Annexe}

Tableau 1 : Les coûts ex ante de transaction

\begin{tabular}{|c|c|c|c|}
\hline Coûts ex ante & $\begin{array}{lr}\text { avant } & 2000 \\
\text { avec } & \text { les } \\
\text { bureaux } & \\
\text { d'affrètement }\end{array}$ & $\begin{array}{l}\text { De } 2000 \text { à } 2007 \text { : } \\
\text { Courtiers aidés de CIS }\end{array}$ & $\begin{array}{l}\text { Depuis 2008 : Courtiers } \\
\text { sans CIS }\end{array}$ \\
\hline $\begin{array}{l}\text { Coût } \\
\text { information et } \\
\text { de Recherche de } \\
\text { la transaction } \\
\text { pour le chargeur }\end{array}$ & inexistant & $\begin{array}{l}1 \text { appel auprès d'un } \\
\text { courtier ou transmission } \\
\text { de la D à CIS ; Facture } \\
\text { téléphonique et temps de } \\
\text { communication }\end{array}$ & $\begin{array}{l}1 \text { ou plusieurs appels de } \\
\text { courtiers/commissionnaires } \\
\text { de transport }\end{array}$ \\
\hline $\begin{array}{l}\text { Coût } \\
\text { information et } \\
\text { de Recherche de } \\
\text { la transaction } \\
\text { par le courtier }\end{array}$ & inexistant & $\begin{array}{l}1 \text { appel du courtier auprès } \\
\text { de CIS, et/ou auprès d'un } \\
\text { ou plusieurs bateliers }\end{array}$ & $\begin{array}{l}\text { Temps de recherche d'un } \\
\text { batelier disponible + facture } \\
\text { téléphone }\end{array}$ \\
\hline $\begin{array}{l}\text { Coût } \\
\text { information et } \\
\text { de Recherche de } \\
\text { la transaction } \\
\text { transporteur }\end{array}$ & inexistant & $\begin{array}{l}\text { transmission disponibilité } \\
\text { à CIS; consultation des D } \\
\text { dans l'une des } 6 \text { places de } \\
\text { marché }+ \text { frais de tel } \\
(1,4 \% \text { du CA })\end{array}$ & $\begin{array}{l}\text { appels téléphoniques des } \\
\text { courtiers habituels voire } \\
\text { occasionnels du batelier } \\
(1,8 \% \text { du CA })\end{array}$ \\
\hline $\begin{array}{l}\text { Connaissance de } \\
\text { l'offre et de la } \\
\text { demande (dispo } \\
\text { cale) }\end{array}$ & $\begin{array}{l}\text { Publique : } \\
\text { Liste des O et } \\
\text { des D locales } \\
\text { affichée par le } \\
\text { bureau } \\
\text { d'affrètement }\end{array}$ & $\begin{array}{l}\text { Semi-publique pour les } \\
\text { transactions passant par } \\
\text { CIS : CIS centralisait les } \\
\text { O et les D locales et les } \\
\text { affichait dans ses } 6 \text { salles } \\
\text { de marché }\end{array}$ & $\begin{array}{l}\text { Privée : seuls les courtiers } \\
\text { centralisent localement les } \\
\text { O et D de cale; } \\
\text { connaissance informelle du } \\
\text { marché par les bateliers }\end{array}$ \\
\hline $\begin{array}{l}\text { Déséquilibre } \\
\text { flux et } \\
\text { tarification }\end{array}$ & prix administré & $\begin{array}{l}\text { prix connu via CIS, } \\
\text { méconnu pour les autres } \\
\text { transactions }\end{array}$ & $\begin{array}{l}\text { prix méconnu et prédateur } \\
\text { sur le flux retour }\end{array}$ \\
\hline $\begin{array}{l}\text { Nombre } \\
\text { d'intermédiaires } \\
\text { par transaction }\end{array}$ & 1 & $\begin{array}{l}2 \\
\text { courtier/commissionnaire } \\
\text { et } 1 \text { CIS ) ou } 3 \text { (si le } \\
\text { courtier est différent du } \\
\text { commissionnaire) }\end{array}$ & 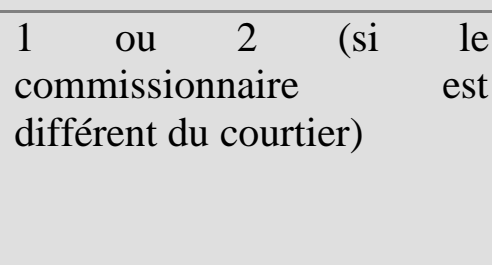 \\
\hline $\begin{array}{l}\text { Coût } \\
\text { d'intermédiation } \\
\text { pour le batelier } \\
\text { en } \% \text { de la }\end{array}$ & $6,25 \%$ & 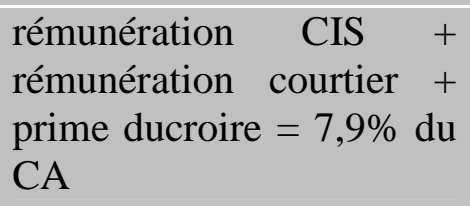 & $\begin{array}{l}\text { Rémunération du courtier }+ \\
\text { prime durcroire }=6,3 \% \text { du } \\
\text { CA }\end{array}$ \\
\hline
\end{tabular}




\begin{tabular}{|c|c|c|c|}
\hline transaction & & & \\
\hline $\begin{array}{l}\text { Coût négociation } \\
\text { des termes du } \\
\text { contrat }\end{array}$ & $\begin{array}{l}\text { Nul, car prix } \\
\text { administré }\end{array}$ & $\begin{array}{l}\text { dépendant du nombre de } \\
\text { bateliers sollicités }\end{array}$ & $\begin{array}{l}\text { dépendant du nombre de } \\
\text { bateliers sollicités }\end{array}$ \\
\hline $\begin{array}{l}\text { Coût de } \\
\text { coordination } \\
\text { (organisation des } \\
\text { affrètements) }\end{array}$ & $\begin{array}{l}\text { Mutualisé et } \\
\text { supporté par le } \\
\text { bureau } \\
\text { d'affrètement }\end{array}$ & $\begin{array}{l}\text { Mutualisé et supporté par } \\
\text { CIS et les courtiers }\end{array}$ & $\begin{array}{l}\text { Supporté par le batelier } \\
\text { rémunérant un ou plusieurs } \\
\text { courtiers }\end{array}$ \\
\hline $\begin{array}{ll}\text { Coût } & \text { de } \\
\text { rédaction et } & \text { de } \\
\text { conclusion } & \text { du } \\
\text { contrat } & \end{array}$ & $\begin{array}{l}\text { nul car } \\
\text { administré }\end{array}$ & $\begin{array}{l}\text { faible mais avec des frais } \\
\text { de dossier variables entre } \\
5 \text { et } 22 € \text { à la charge des } \\
\text { transporteurs }\end{array}$ & $\begin{array}{l}\text { faible, car souvent conclu } \\
\text { oralement, avec des frais de } \\
\text { dossier variables entre } 5 \text { et } \\
22 € \text { à la charge des } \\
\text { transporteurs }\end{array}$ \\
\hline $\begin{array}{l}\text { Total des coûts } \\
\text { ex ante : }\end{array}$ & -- & + & ++ \\
\hline
\end{tabular}

